\title{
Dirac operators on noncommutative hypersurfaces
}

\author{
Hans Nguyen ${ }^{a}$ and Alexander Schenkel ${ }^{b}$ \\ School of Mathematical Sciences, University of Nottingham, \\ University Park, Nottingham NG7 2RD, United Kingdom. \\ Email: ${ }^{a}$ hans.nguyen@nottingham.ac.uk, ${ }^{b}$ alexander.schenkel@nottingham.ac.uk
}

August 2020

\begin{abstract}
This paper studies geometric structures on noncommutative hypersurfaces within a moduletheoretic approach to noncommutative Riemannian (spin) geometry. A construction to induce differential, Riemannian and spinorial structures from a noncommutative embedding space to a noncommutative hypersurface is developed and applied to obtain noncommutative hypersurface Dirac operators. The general construction is illustrated by studying the sequence $\mathbb{T}_{\theta}^{2} \hookrightarrow \mathbb{S}_{\theta}^{3} \hookrightarrow \mathbb{R}_{\theta}^{4}$ of noncommutative hypersurface embeddings.
\end{abstract}

Keywords: noncommutative geometry, bimodule connections, Dirac operators, noncommutative hypersurfaces

MSC 2010: $81 \mathrm{~T} 75,81 \mathrm{R} 50,46 \mathrm{~L} 87$

\section{Contents}

\begin{tabular}{lll}
\hline & Introduction and summary & 1
\end{tabular}

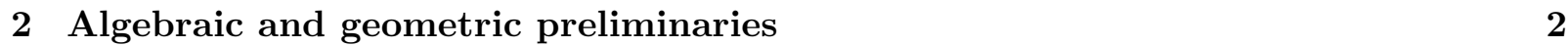

3 Induced structures on noncommutative hypersurfaces 5

$3.1 \quad$ Noncommutative hypersurfaces $\ldots \ldots \ldots \ldots \ldots \ldots \ldots$

3.2 Induced Riemannian structure $\ldots \ldots \ldots \ldots \ldots \ldots$

$3.3 \quad$ Induced spinorial structure $\ldots \ldots \ldots \ldots \ldots \ldots$. . . . . . . . . . . . . . 10

$\begin{array}{lll}4 & \text { Examples } & 11\end{array}$

4.1 Noncommutative embedding space $\mathbb{R}_{\theta}^{4} \ldots \ldots \ldots \ldots \ldots \ldots \ldots$. . . . . . . . . 12

$4.2 \quad$ Noncommutative hypersurface $\mathbb{S}_{\theta}^{3} \hookrightarrow \mathbb{R}_{\theta}^{4} \ldots \ldots \ldots \ldots \ldots \ldots \ldots \ldots \ldots$

$4.3 \quad$ Noncommutative hypersurface $\mathbb{T}_{\theta}^{2} \hookrightarrow \mathbb{S}_{\theta}^{3} \ldots \ldots \ldots \ldots \ldots \ldots \ldots$

\section{Introduction and summary}

Dirac operators play a fundamental role in both quantum physics and noncommutative geometry. From the point of view of Connes' axiomatization of noncommutative Riemannian spin manifolds in terms of spectral triples [Con94, a Dirac operator is the basic object that is supposed to encode all geometric information about the noncommutative space. However, the way in which a Dirac operator encodes this geometric data is rather implicit, hence it is in general difficult to extract information about the metric or curvature of a noncommutative space, see e.g. [CM14]. 
An alternative approach to noncommutative Riemannian (spin) geometry is to encode the relevant geometric data layer by layer in terms of noncommutative generalizations of differential calculi, metrics, connections and spinorial structures, see e.g. [Lan97, [D-V01] and [BM20]. This module-theoretic approach maintains closer ties to the structures familiar from classical differential geometry, which can be very beneficial for constructing, analyzing and also interpreting examples of noncommutative spaces. Moreover, due to results by Beggs and Majid [BM17], this approach leads under certain additional hypotheses to examples of spectral triples in the sense of Con94.

The aim of the present paper is to develop techniques that allow us to induce differential, Riemannian and spinorial structures from a noncommutative embedding space to a noncommutative hypersurface. Our construction is a noncommutative generalization of well-known results in classical differential geometry, see e.g. [Bur93, Tra95, Bar96, HMZ02, and it results in an explicit formula for the Dirac operator on the noncommutative hypersurface. In particular, our techniques and results can be applied to construct examples of curved noncommutative hypersurfaces and their Dirac operators from very simple flat noncommutative embedding spaces.

The outline of the remainder of this paper is as follows: In Section 2 we provide a brief review of the relevant algebraic and geometric preliminaries from the module-theoretic approach to noncommutative Riemannian (spin) geometry. Section 3 presents our main results on induced differential, Riemannian and spinorial structures on noncommutative hypersurfaces in the sense of Definition 3.2. Our construction requires certain additional hypotheses on the structure of the noncommutative hypersurface under consideration, which we will introduce consecutively as soon as they are needed. We refer the reader to Assumptions 3.6, 3.10 and 3.12 for a complete list of these hypotheses. The main result of this section is Proposition 3.14, where we derive an explicit expression for the Dirac operator on the noncommutative hypersurface. In Section 4 we illustrate our constructions and results by applying them to the sequence of noncommutative hypersurface embeddings $\mathbb{T}_{\theta}^{2} \hookrightarrow \mathbb{S}_{\theta}^{3} \hookrightarrow \mathbb{R}_{\theta}^{4}$ studied by Arnlind and Norkvist [AN19. Starting from a very simple flat noncommutative geometry on $\mathbb{R}_{\theta}^{4}$, we compute the induced geometric structures on both the Connes-Landi sphere $\mathbb{S}_{\theta}^{3}$ and the noncommutative torus $\mathbb{T}_{\theta}^{2}$. Our induced hypersurface Dirac operators on $\mathbb{S}_{\theta}^{3}$ and $\mathbb{T}_{\theta}^{2}$ are isospectral to the commutative ones and they coincide with the Dirac operators obtained from toric deformations in [CL01, CD-V02, BLvS13].

\section{Algebraic and geometric preliminaries}

In this paper all vector spaces, algebras, modules, etc., will be over the field $\mathbb{C}$ of complex numbers. Given an (associative and unital) algebra $A$, we denote by ${ }_{A}$ Mod the category of left $A$-modules and by ${ }_{A} \operatorname{Mod}_{A}$ the category of $A$-bimodules. Recall that the latter category is monoidal with respect to the relative tensor product $V \otimes_{A} W \in{ }_{A} \operatorname{Mod}_{A}$ of $A$-bimodules $V, W \in{ }_{A} \operatorname{Mod}_{A}$ and monoidal unit given by the 1-dimensional free $A$-bimodule $A \in{ }_{A} \operatorname{Mod}_{A}$. Furthermore, ${ }_{A}$ Mod is a (left) module category over the monoidal category $\left({ }_{A} \operatorname{Mod}_{A}, \otimes_{A}, A\right)$, with left action given by the relative tensor product $V \otimes_{A} \mathcal{E} \in{ }_{A} \operatorname{Mod}$, for all $V \in{ }_{A} \operatorname{Mod}_{A}$ and $\mathcal{E} \in{ }_{A} \operatorname{Mod}$.

Let us recall briefly some basic concepts from noncommutative geometry, see e.g. [Lan97, [D-V01] and [BM20] for a detailed introduction to the relevant frameworks.

Definition 2.1. A (first-order) differential calculus on an algebra $A$ is a pair $\left(\Omega_{A}^{1}, \mathrm{~d}\right)$ consisting of an $A$-bimodule $\Omega_{A}^{1} \in{ }_{A} \operatorname{Mod}_{A}$ and a linear map d $: A \rightarrow \Omega_{A}^{1}$ (called differential), such that

(i) $\mathrm{d}\left(a a^{\prime}\right)=(\mathrm{d} a) a^{\prime}+a\left(\mathrm{~d} a^{\prime}\right)$, for all $a, a^{\prime} \in A$,

(ii) $\Omega_{A}^{1}=A \mathrm{~d}(A):=\left\{\sum_{i} a_{i} \mathrm{~d} a_{i}^{\prime}: a_{i}, a_{i}^{\prime} \in A\right\}$.

We call $\Omega_{A}^{1}$ the $A$-bimodule of 1-forms on $A$.

Definition 2.2. Let $\left(\Omega_{A}^{1}, \mathrm{~d}\right)$ be a differential calculus on an algebra $A$. 
(i) A connection on a left $A$-module $\mathcal{E} \in{ }_{A}$ Mod is a linear map $\nabla: \mathcal{E} \rightarrow \Omega_{A}^{1} \otimes_{A} \mathcal{E}$ that satisfies the left Leibniz rule

$$
\nabla(a s)=a \nabla(s)+\mathrm{d} a \otimes_{A} s,
$$

for all $a \in A$ and $s \in \mathcal{E}$.

(ii) A bimodule connection on an $A$-bimodule $V \in{ }_{A} \operatorname{Mod}_{A}$ is a pair $(\nabla, \sigma)$ consisting of a linear map $\nabla: V \rightarrow \Omega_{A}^{1} \otimes_{A} V$ and an $A$-bimodule isomorphism $\sigma: V \otimes_{A} \Omega_{A}^{1} \rightarrow \Omega_{A}^{1} \otimes_{A} V$, such that the following left and right Leibniz rules

$$
\begin{aligned}
& \nabla(a v)=a \nabla(v)+\mathrm{d} a \otimes_{A} v, \\
& \nabla(v a)=\nabla(v) a+\sigma\left(v \otimes_{A} \mathrm{~d} a\right),
\end{aligned}
$$

are satisfied, for all $a \in A$ and $v \in V$.

The concept of bimodule connections is motivated by the following standard result, see e.g. [D-V01, Section 10].

Proposition 2.3. Let $\left(\Omega_{A}^{1}\right.$, d) be a differential calculus on an algebra $A$.

(i) Let $\nabla^{\mathcal{E}}$ be a connection on a left $A$-module $\mathcal{E} \in{ }_{A} \operatorname{Mod}$ and $\left(\nabla^{V}, \sigma^{V}\right)$ a bimodule connection on an A-bimodule $V \in{ }_{A} \operatorname{Mod}_{A}$. Then

$$
\nabla^{\otimes}\left(v \otimes_{A} s\right):=\nabla^{V}(v) \otimes_{A} s+\left(\sigma^{V} \otimes_{A} \mathrm{id}\right)\left(v \otimes_{A} \nabla^{\mathcal{E}}(s)\right),
$$

for all $v \in V$ and $s \in \mathcal{E}$, defines a connection on the tensor product module $V \otimes_{A} \mathcal{E} \in{ }_{A}$ Mod.

(ii) Let $\left(\nabla^{V}, \sigma^{V}\right)$ and $\left(\nabla^{W}, \sigma^{W}\right)$ be bimodule connections on two A-bimodules $V, W \in{ }_{A} \operatorname{Mod}_{A}$. Then

$$
\nabla^{\otimes}\left(v \otimes_{A} w\right):=\nabla^{V}(v) \otimes_{A} w+\left(\sigma^{V} \otimes_{A} \mathrm{id}\right)\left(v \otimes_{A} \nabla^{W}(w)\right)
$$

for all $v \in V$ and $w \in W$, and the composite A-bimodule isomorphism

$$
\sigma^{\otimes}: V \otimes_{A} W \otimes_{A} \Omega_{A}^{1} \stackrel{\mathrm{id} \otimes_{A} \sigma^{W}}{\longrightarrow} V \otimes_{A} \Omega_{A}^{1} \otimes_{A} W \stackrel{\sigma^{V} \otimes_{A} \mathrm{id}}{\longrightarrow} \Omega_{A}^{1} \otimes_{A} V \otimes_{A} W
$$

defines a bimodule connection on the tensor product bimodule $V \otimes_{A} W \in{ }_{A} \operatorname{Mod}_{A}$.

Definition 2.4. A (generalized) metric on $\Omega_{A}^{1}$ is an $A$-bimodule map $g: A \rightarrow \Omega_{A}^{1} \otimes_{A} \Omega_{A}^{1}$ for which there exists an $A$-bimodule map $g^{-1}: \Omega_{A}^{1} \otimes_{A} \Omega_{A}^{1} \rightarrow A$, such that the two compositions

$$
\begin{aligned}
& \Omega_{A}^{1} \cong \Omega_{A}^{1} \otimes_{A} A \stackrel{\mathrm{id} \otimes_{A} g}{\longrightarrow} \Omega_{A}^{1} \otimes_{A} \Omega_{A}^{1} \otimes_{A} \Omega_{A}^{1} \stackrel{g^{-1} \otimes_{A} \mathrm{id}}{\longrightarrow} A \otimes_{A} \Omega_{A}^{1} \cong \Omega_{A}^{1} \\
& \Omega_{A}^{1} \cong A \otimes_{A} \Omega_{A}^{1} \stackrel{g \otimes_{A} \mathrm{id}}{\longrightarrow} \Omega_{A}^{1} \otimes_{A} \Omega_{A}^{1} \otimes_{A} \Omega_{A}^{1} \stackrel{\mathrm{id} \otimes_{A} g^{-1}}{\longrightarrow} \Omega_{A}^{1} \otimes_{A} A \cong \Omega_{A}^{1}
\end{aligned}
$$

are the identity morphisms. We call $g^{-1}$ the inverse metric.

Remark 2.5. Since $A$ is a free module with a basis given by the unit element $1 \in A$, the datum of an $A$-bimodule map $g: A \rightarrow \Omega_{A}^{1} \otimes_{A} \Omega_{A}^{1}$ is equivalent to that of a central element $g(1) \in \Omega_{A}^{1} \otimes_{A} \Omega_{A}^{1}$, i.e. $a g(1)=g(1) a$ for all $a \in A$. Writing this element as $g(1)=\sum_{\alpha} g^{\alpha} \otimes_{A} g_{\alpha}$, the two conditions in $(2.5)$ read as

$$
\sum_{\alpha} g^{-1}\left(\omega \otimes_{A} g^{\alpha}\right) g_{\alpha}=\omega=\sum_{\alpha} g^{\alpha} g^{-1}\left(g_{\alpha} \otimes_{A} \omega\right)
$$

for all $\omega \in \Omega_{A}^{1}$. Using these identities it is easy to prove that, provided it exists, the inverse metric $g^{-1}$ is unique. 
Definition 2.6. Let $\left(\Omega_{A}^{1}, \mathrm{~d}\right)$ be a differential calculus on an algebra A. A Riemannian structure on $\left(\Omega_{A}^{1}, \mathrm{~d}\right)$ is a pair $(g,(\nabla, \sigma))$ consisting of a (generalized) metric $g$ on $\Omega_{A}^{1}$ and a bimodule connection $(\nabla, \sigma)$ on $\Omega_{A}^{1}$ that satisfies the following properties:

(i) Symmetry: The diagram

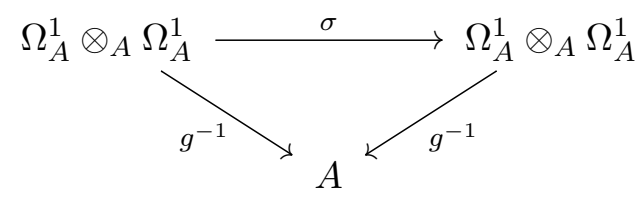

commutes.

(ii) Metric compatibility: The diagram

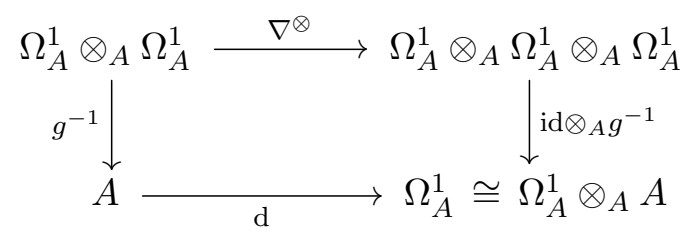

commutes, where $\nabla^{\otimes}$ is the tensor product connection from Proposition 2.3 .

Remark 2.7. Note that our definition of Riemannian structures does not include a torsionfree condition for the connection $\nabla$. In those cases where one has a second-order differential calculus $\Omega_{A}^{2}$, one can supplement Definition 2.6 with the torsion-free condition $T=0$, where $T:=\wedge \circ \nabla-\mathrm{d}: \Omega_{A}^{1} \rightarrow \Omega_{A}^{2}$ is the torsion tensor, see [BM20]. The reason why we do not consider the torsion-free condition is that our constructions in this paper apply to connections with torsion too, hence this condition is not needed.

Next, we introduce a suitable concept of spinorial structure based on the module-theoretic approach by Beggs and Majid [BM17, BM20]. Let $\left(\Omega_{A}^{1}, \mathrm{~d}\right)$ be a differential calculus on an algebra $A$ and $(g,(\nabla, \sigma))$ a Riemannian structure on $\left(\Omega_{A}^{1}, \mathrm{~d}\right)$. Consider a left $A$-module $\mathcal{E} \in{ }_{A}$ Mod, which we interpret as the module of sections of a spinor bundle. This module should come endowed with a connection $\nabla^{\mathrm{sp}}: \mathcal{E} \rightarrow \Omega_{A}^{1} \otimes_{A} \mathcal{E}$, which we interpret as spin connection, and an $A$-module map $\gamma: \Omega_{A}^{1} \otimes_{A} \mathcal{E} \rightarrow \mathcal{E}$, which we interpret as Clifford multiplication. These data will be required to be compatible (in the sense defined below) with the Riemannian structure $(g,(\nabla, \sigma))$. For later use, let us introduce the notation

$$
\gamma_{[2]}: \Omega_{A}^{1} \otimes_{A} \Omega_{A}^{1} \otimes_{A} \mathcal{E} \stackrel{\operatorname{id} \otimes_{A} \gamma}{\longrightarrow} \Omega_{A}^{1} \otimes_{A} \mathcal{E} \stackrel{\gamma}{\longrightarrow} \mathcal{E}
$$

for the $A$-module map obtained by iterated application of $\gamma$. Analogously, one can define $\gamma_{[n]}$ : $\Omega_{A}^{1 \otimes_{A} n} \otimes_{A} \mathcal{E} \rightarrow \mathcal{E}$, for all $n \in \mathbb{N}$.

Definition 2.8. Let $\left(\Omega_{A}^{1}, \mathrm{~d}\right)$ be a differential calculus on an algebra $A$ and $(g,(\nabla, \sigma))$ a Riemannian structure on $\left(\Omega_{A}^{1}, \mathrm{~d}\right)$. A spinorial structure on $(g,(\nabla, \sigma))$ is a triple $\left(\mathcal{E}, \nabla^{\mathrm{sp}}, \gamma\right)$ consisting of a left $A$-module $\mathcal{E} \in{ }_{A}$ Mod, a connection $\nabla^{\text {sp }}$ on $\mathcal{E}$ and an $A$-module map $\gamma: \Omega_{A}^{1} \otimes_{A} \mathcal{E} \rightarrow \mathcal{E}$ that satisfies the following properties:

(i) Clifford relations: The diagram

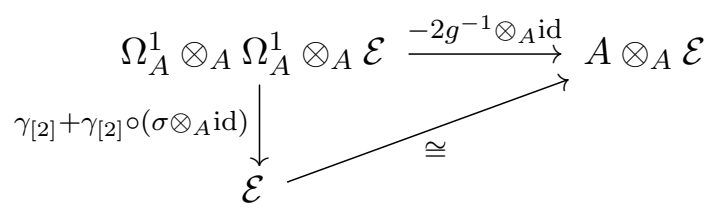

commutes. 
(ii) Clifford compatibility: The diagram

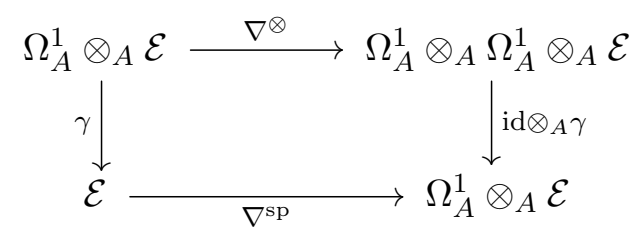

commutes, where $\nabla^{\otimes}$ is the tensor product connection from Proposition 2.3

We shall call the composite

$$
D: \mathcal{E} \stackrel{\nabla^{\mathrm{sp}}}{\longrightarrow} \Omega_{A}^{1} \otimes_{A} \mathcal{E} \stackrel{\gamma}{\longrightarrow} \mathcal{E}
$$

the Dirac operator associated with the given spinorial structure.

For later use, we record the following property of the Dirac operator.

Proposition 2.9. The Dirac operator 2.12 satisfies

$$
D(a s)=a D(s)+\gamma\left(\mathrm{d} a \otimes_{A} s\right),
$$

for all $a \in A$ and $s \in \mathcal{E}$.

Proof. This is a direct consequence of the Leibniz rule (2.1) for $\nabla^{\text {sp }}$ and the fact that $\gamma$ is left A-linear.

Remark 2.10. We would like to emphasize that our definition of spinorial structures is less general than the one by Beggs and Majid [BM17, BM20, which does not assume Clifford compatibility (2.11). We decided to include this additional axiom in our definition, because it is an important guiding principle for our construction of Dirac operators on noncommutative hypersurfaces in Section 3 and it is satisfied in our examples of interest in Section 4 .

\section{Induced structures on noncommutative hypersurfaces}

Throughout the whole section, we fix an algebra $A$, a differential calculus $\left(\Omega_{A}^{1}\right.$, d) on $A$ (see Definition 2.1), a Riemannian structure $(g,(\nabla, \sigma))$ on $\left(\Omega_{A}^{1}, \mathrm{~d}\right)$ (see Definition 2.6) and a spinorial structure $\left(\mathcal{E}, \nabla^{\mathrm{sp}}, \gamma\right)$ on $(g,(\nabla, \sigma))$ (see Definition 2.8). We interpret $A$ as (the algebra of functions on) a noncommutative embedding space, which is endowed with a differential, Riemannian and spinorial structure.

\subsection{Noncommutative hypersurfaces}

In this subsection we introduce a suitable class of noncommutative hypersurfaces that will form the basis for our studies. Given a 2-sided ideal $I \subset A$, consider the quotient algebra

$$
B:=A / I
$$

Associated with the quotient algebra map $q: A \rightarrow B$ is a change of base functor $q_{!}:{ }_{A} \operatorname{Mod}_{A} \rightarrow$ ${ }_{B} \operatorname{Mod}_{B}$ for bimodules, which is given by $q !(V)=B \otimes_{A} V \otimes_{A} B \in{ }_{B} \operatorname{Mod}_{B}$, for all $V \in{ }_{A} \operatorname{Mod}_{A}$. Because $B=A / I$ is a quotient algebra and $q: A \rightarrow B$ is the corresponding quotient map, there exists a natural $B$-bimodule isomorphism

$$
q_{!}(V) \stackrel{V}{\longrightarrow} \frac{V}{I V \cup V I}, \quad[a] \otimes_{A} v \otimes_{A}\left[a^{\prime}\right] \longmapsto\left[a v a^{\prime}\right],
$$


where $I V:=\{a v: a \in I$ and $v \in V\} \subseteq V$ and $V I:=\{v a: a \in I$ and $v \in V\} \subseteq V$. Here and in the following, we use square brackets to denote equivalence classes in quotient spaces. Applying the change of base functor on the $A$-bimodule of 1 -forms $\Omega_{A}^{1} \in{ }_{A} \operatorname{Mod}_{A}$ is however not sufficient to define a differential calculus on $B$, because the differential d : $A \rightarrow q_{!}\left(\Omega_{A}^{1}\right)$ does not in general descend to the quotient $B=A / I$. Following e.g. [BM20, Exercise E1.4], this problem is solved by introducing the quotient $B$-bimodule

$$
\Omega_{B}^{1}:=\frac{q_{!}\left(\Omega_{A}^{1}\right)}{B[\mathrm{~d} I] B} \in{ }_{B} \operatorname{Mod}_{B} \quad,
$$

where $B[\mathrm{~d} I] B:=\left\{\sum_{i} b_{i}\left[\mathrm{~d} a_{i}\right] b_{i}^{\prime}: b_{i}, b_{i}^{\prime} \in B\right.$ and $\left.a_{i} \in I\right\}$ is the $B$-subbimodule generated by $[\mathrm{d} I] \subseteq q_{!}\left(\Omega_{A}^{1}\right)$. The differential $\mathrm{d}: A \rightarrow \Omega_{A}^{1}$ then descends to a linear map

$$
\mathrm{d}_{B}: B \longrightarrow \Omega_{B}^{1}, \quad[a] \longmapsto[\mathrm{d} a]
$$

and we obtain

Proposition 3.1. $\left(\Omega_{B}^{1}, \mathrm{~d}_{B}\right)$ is a differential calculus on the quotient algebra $B=A / I$.

Proof. The necessary properties of Definition 2.1 are inherited from the differential calculus $\left(\Omega_{A}^{1}, \mathrm{~d}\right)$ on $A$, see e.g. [BM20, Exercise E1.4].

The scenario introduced above is too general to interpret $q: A \rightarrow B$ as (the dual of) an embedding of a noncommutative hypersurface $B$ into the noncommutative embedding space $A$. In particular, it does not capture that $B$ should be of "codimension 1" and that we would like the existence of a "normalized normal vector field" for $B$. In order to introduce an appropriate noncommutative generalization of these concepts 1 , we note that the canonical quotient map $q_{!}\left(\Omega_{A}^{1}\right) \rightarrow \Omega_{B}^{1}$ (cf. (3.3)) gives rise to a short exact sequence of $B$-bimodules

$$
0 \longrightarrow N_{B}^{1}:=B[\mathrm{~d} I] B \longrightarrow q_{!}\left(\Omega_{A}^{1}\right) \longrightarrow \Omega_{B}^{1} \longrightarrow 0
$$

where $N_{B}^{1} \in{ }_{B} \operatorname{Mod}_{B}$ is a noncommutative analogue of the conormal bundle.

Definition 3.2. We say that $B=A / I$ is a (metrically co-orientable) noncommutative hypersurface if the $B$-bimodule $N_{B}^{1} \in{ }_{B} \operatorname{Mod}_{B}$ admits a 1-dimensional basis $[\nu] \in N_{B}^{1}$ with $\nu \in \Omega_{A}^{1}$ a central 1-form, i.e. $a \nu=\nu a$ for all $a \in A$, that satisfies the normalization condition

$$
\left[g^{-1}\left(\nu \otimes_{A} \nu\right)\right]=1 \in B
$$

Remark 3.3. Definition 3.2 captures both the property of $B$ being of "codimension 1", which is encoded by the statement that $N_{B}^{1}$ has rank 1 , and the existence of a "normalized normal vector field", which in our dual language of forms is given by the normalized $\nu \in \Omega_{A}^{1}$. Throughout this paper, we shall always use the simpler terminology of noncommutative hypersurfaces instead of the technically more appropriate, but cumbersome, term metrically co-orientable noncommutative hypersurfaces that emphasises existence of the normalized 1-form $\nu$.

Example 3.4. An important and interesting class of examples of noncommutative hypersurfaces in the sense of Definition 3.2 is given by noncommutative level set hypersurfaces. These are determined by 2 -sided ideals $I=(f) \subset A$ generated by a central element $f \in \mathcal{Z}(A) \subseteq A$ such that $\nu:=\mathrm{d} f \in \Omega_{A}^{1}$ is central and satisfies the normalization condition $(3.6)$. It is easy to see that in this case $[\nu]=[\mathrm{d} f]$ defines a basis of $N_{B}^{1}=B[\mathrm{~d} I] B=B[\mathrm{~d} f] B=[\mathrm{d} f] B=B[\mathrm{~d} f]$, where in the last two steps we used that $\mathrm{d} f$ is central. All our examples in Section 4 will be of this type. $\nabla$

\footnotetext{
${ }^{1}$ We would like to thank Branimir Ćaćić for suggesting Definition 3.2 to us. This allowed us to generalize our results for noncommutative level set hypersurfaces (cf. Example 3.4 from the first version of this paper.
} 
In what follows we let $B=A / I$ be any noncommutative hypersurface in the sense of Definition 3.2. As a preparation for the following subsections, we construct a splitting of the sequence (3.5) that determines a $B$-bimodule isomorphism between $\Omega_{B}^{1} \in{ }_{B} \operatorname{Mod}_{B}$ and a certain $B$-subbimodule of $q_{!}\left(\Omega_{A}^{1}\right) \in{ }_{B} \operatorname{Mod}_{B}$. Using the inverse metric $g^{-1}: \Omega_{A}^{1} \otimes_{A} \Omega_{A}^{1} \rightarrow A$ and the normalized 1-form $\nu \in \Omega_{A}^{1}$, we define the $A$-bimodule endomorphism

$$
\Pi: \Omega_{A}^{1} \longrightarrow \Omega_{A}^{1}, \omega \longmapsto \omega-g^{-1}\left(\omega \otimes_{A} \nu\right) \nu .
$$

Note that $\Pi$ is indeed right $A$-linear because $\nu$ is by hypothesis central. Using the change of base functor, $\Pi$ defines a $B$-bimodule endomorphism

$$
\Pi: q_{!}\left(\Omega_{A}^{1}\right) \longrightarrow q_{!}\left(\Omega_{A}^{1}\right), \quad[\omega] \longmapsto \Pi([\omega]):=[\Pi(\omega)] .
$$

Proposition 3.5. The B-bimodule endomorphism $\Pi$ from (3.7b) satisfies the following properties:

(i) $\Pi([\nu])=0$.

(ii) $\Pi^{2}=\Pi$, i.e. $\Pi$ is a projector.

(iii) The induced B-bimodule map $\Pi: \Omega_{B}^{1} \rightarrow q_{!}\left(\Omega_{A}^{1}\right)$ on $\Omega_{B}^{1}$ (cf. (3.3)) is a section of the quotient $B$-bimodule map $q_{!}\left(\Omega_{A}^{1}\right) \rightarrow \Omega_{B}^{1}$. In particular, it defines an isomorphism $\Omega_{B}^{1} \cong \Pi q_{!}\left(\Omega_{A}^{1}\right)$.

Proof. Item (i) follows directly from the normalization condition (3.6) and item (ii) is a direct consequence of (i). To prove item (iii), note that the induced map $\Pi: \Omega_{B}^{1} \rightarrow q_{!}\left(\Omega_{A}^{1}\right)$ is welldefined because of (i) and the fact that $[\nu]$ is by hypothesis a basis for $N_{B}^{1}$. It is a section of the quotient map because the latter maps $[\nu]$ to 0 . This in particular implies that the induced map $\Pi: \Omega_{B}^{1} \rightarrow q_{!}\left(\Omega_{A}^{1}\right)$ is injective, hence it defines an isomorphism onto its image $\Pi q_{!}\left(\Omega_{A}^{1}\right)$.

\subsection{Induced Riemannian structure}

The aim of this subsection is to induce a Riemannian structure $\left(g_{B},\left(\nabla_{B}, \sigma_{B}\right)\right)$ on the differential calculus $\left(\Omega_{B}^{1}\right.$,d) from Section 3.1. Using the change of base functor, the metric $g: A \rightarrow \Omega_{A}^{1} \otimes_{A} \Omega_{A}^{1}$ on $\Omega_{A}^{1}$ defines a $B$-bimodule map

$$
g: B \longrightarrow q_{!}\left(\Omega_{A}^{1}\right) \otimes_{B} q_{!}\left(\Omega_{A}^{1}\right), \quad[a] \longmapsto[g(a)]
$$

and the inverse metric $g^{-1}: \Omega_{A}^{1} \otimes_{A} \Omega_{A}^{1} \rightarrow A$ defines a $B$-bimodule map

$$
g^{-1}: q_{!}\left(\Omega_{A}^{1}\right) \otimes_{B} q_{!}\left(\Omega_{A}^{1}\right) \longrightarrow B,[\omega] \otimes_{B}[\zeta] \longmapsto\left[g^{-1}\left(\omega \otimes_{A} \zeta\right)\right] .
$$

Using also the quotient map $q_{!}\left(\Omega_{A}^{1}\right) \rightarrow \Omega_{B}^{1}$ and its section $\Pi: \Omega_{B}^{1} \rightarrow q_{!}\left(\Omega_{A}^{1}\right)$ from Proposition 3.5 (see also (3.7)), we define the composite $B$-bimodule maps

$$
g_{B}: \quad B \stackrel{g}{\longrightarrow} q_{!}\left(\Omega_{A}^{1}\right) \otimes_{B} q_{!}\left(\Omega_{A}^{1}\right) \longrightarrow \Omega_{B}^{1} \otimes_{B} \Omega_{B}^{1}
$$

and

$$
g_{B}^{-1}: \Omega_{B}^{1} \otimes_{B} \Omega_{B}^{1} \stackrel{\Pi \otimes_{B} \Pi}{\longrightarrow} q_{!}\left(\Omega_{A}^{1}\right) \otimes_{B} q_{!}\left(\Omega_{A}^{1}\right) \stackrel{g^{-1}}{\longrightarrow} B .
$$

For our studies below, we shall need the following

Assumption 3.6. The $A$-bimodule isomorphism $\sigma: \Omega_{A}^{1} \otimes_{A} \Omega_{A}^{1} \rightarrow \Omega_{A}^{1} \otimes_{A} \Omega_{A}^{1}$ associated with the bimodule connection $(\nabla, \sigma)$ on $\Omega_{A}^{1}$ satisfies

$$
\sigma\left(\omega \otimes_{A} \nu\right)=\nu \otimes_{A} \omega, \quad \sigma\left(\nu \otimes_{A} \omega\right)=\omega \otimes_{A} \nu,
$$

for all $\omega \in \Omega_{A}^{1}$. 
Lemma 3.7. Assumption 3.6 implies the following properties:

(i) $g^{-1}\left(\omega \otimes_{A} \nu\right)=g^{-1}\left(\nu \otimes_{A} \omega\right)$, for all $\omega \in \Omega_{A}^{1}$.

(ii) $\left[g^{-1}\left(\Pi(\omega) \otimes_{A} \nu\right)\right]=0$ and $\left[g^{-1}\left(\nu \otimes_{A} \Pi(\omega)\right)\right]=0$ in $B=A / I$, for all $\omega \in \Omega_{A}^{1}$. This implies that

$$
\left[g^{-1}\left(\Pi(\omega) \otimes_{A} \Pi(\zeta)\right)\right]=\left[g^{-1}\left(\omega \otimes_{A} \Pi(\zeta)\right)\right]=\left[g^{-1}\left(\Pi(\omega) \otimes_{A} \zeta\right)\right]
$$

for all $\omega, \zeta \in \Omega_{A}^{1}$.

(iii) $\left[\left(\mathrm{id} \otimes_{A} g^{-1}\right)\left(\nabla(\nu) \otimes_{A} \nu\right)\right]=0$.

(iv) The two B-bimodule maps in (3.9) define a metric $g_{B}$ and its inverse $g_{B}^{-1}$ on $\Omega_{B}^{1}$.

Proof. Item (i) is a direct consequence of the symmetry property of $g^{-1}$ (cf. Definition 2.6) and Assumption 3.6. The first equality of item (ii) follows from a short calculation

$$
\begin{aligned}
{\left[g^{-1}\left(\Pi(\omega) \otimes_{A} \nu\right)\right] } & =\left[g^{-1}\left(\left(\omega-g^{-1}\left(\omega \otimes_{A} \nu\right) \nu\right) \otimes_{A} \nu\right)\right] \\
& =\left[g^{-1}\left(\omega \otimes_{A} \nu\right)-g^{-1}\left(\omega \otimes_{A} \nu\right) g^{-1}\left(\nu \otimes_{A} \nu\right)\right]=0,
\end{aligned}
$$

where we used the normalization condition (3.6) for $\nu$. The second equality in item (ii) follows from this and (i).

Item (iii) follows from the calculation

$$
\begin{aligned}
{\left[\left(\mathrm{id} \otimes_{A} g^{-1}\right)\left(\nabla(\nu) \otimes_{A} \nu\right)\right] } & =\left[\mathrm{d}\left(g^{-1}\left(\nu \otimes_{A} \nu\right)\right)-\left(\mathrm{id} \otimes_{A} g^{-1}\right)\left(\sigma \otimes_{A} \mathrm{id}\right)\left(\nu \otimes_{A} \nabla(\nu)\right)\right] \\
& =-\left[\left(\operatorname{id} \otimes_{A} g^{-1}\right)\left(\nabla(\nu) \otimes_{A} \nu\right)\right],
\end{aligned}
$$

where in the first step we used metric compatibility $(2.8)$ and in the second step we used the normalization condition (3.6) and item (i).

To prove item (iv), we use the same notations as in Remark 2.5 to write $g_{B}(1)=[g(1)]=$ $\left[\sum_{\alpha} g^{\alpha} \otimes_{A} g_{\alpha}\right]=\sum_{\alpha}\left[g^{\alpha}\right] \otimes_{B}\left[g_{\alpha}\right]$ and $g_{B}^{-1}\left([\omega] \otimes_{B}[\zeta]\right)=\left[g^{-1}\left(\Pi(\omega) \otimes_{A} \Pi(\zeta)\right)\right]$. We then compute

$$
\sum_{\alpha} g_{B}^{-1}\left([\omega] \otimes_{B}\left[g^{\alpha}\right]\right)\left[g_{\alpha}\right]=\left[\sum_{\alpha} g^{-1}\left(\Pi(\omega) \otimes_{A} g^{\alpha}\right) g_{\alpha}\right]=[\Pi(\omega)]=[\omega] \quad,
$$

where in the first step we used (ii). The second step follows from $g^{-1}$ being the inverse metric of $g$ and the last step uses that $\Pi$ is a section of the quotient map (cf. Proposition 3.5). The second property $\sum_{\alpha}\left[g^{\alpha}\right] g_{B}^{-1}\left(\left[g_{\alpha}\right] \otimes_{B}[\omega]\right)=[\omega]$ follows from a similar calculation.

Let us now focus on the bimodule connection $(\nabla, \sigma)$ on $\Omega_{A}^{1}$. From $(3.3)$, we observe that the connection $\nabla: \Omega_{A}^{1} \rightarrow \Omega_{A}^{1} \otimes_{A} \Omega_{A}^{1}$ descends to a connection $\nabla: q_{!}\left(\Omega_{A}^{1}\right) \rightarrow \Omega_{B}^{1} \otimes_{B} q_{!}\left(\Omega_{A}^{1}\right)$ on $q_{!}\left(\Omega_{A}^{1}\right) \in$ ${ }_{B} \operatorname{Mod}_{B}$. Indeed, from the left Leibniz rule we conclude that $[\nabla(a \omega)]=\left[a \nabla(\omega)+\mathrm{d} a \otimes_{A} \omega\right]=0$, for all $a \in I$, hence this map is well-defined on the quotient. Using the quotient map $q_{!}\left(\Omega_{A}^{1}\right) \rightarrow \Omega_{B}^{1}$ and its section $\Pi: \Omega_{B}^{1} \rightarrow q_{!}\left(\Omega_{A}^{1}\right)$ from Proposition 3.5 (see also (3.7)), we define the composite linear map

$$
\nabla_{B}: \Omega_{B}^{1} \stackrel{\Pi}{\longrightarrow} q_{!}\left(\Omega_{A}^{1}\right) \stackrel{\nabla}{\longrightarrow} \Omega_{B}^{1} \otimes_{B} q_{!}\left(\Omega_{A}^{1}\right) \longrightarrow \Omega_{B}^{1} \otimes_{B} \Omega_{B}^{1} .
$$

The $A$-bimodule isomorphism $\sigma: \Omega_{A}^{1} \otimes_{A} \Omega_{A}^{1} \rightarrow \Omega_{A}^{1} \otimes_{A} \Omega_{A}^{1}$ associated with the bimodule connection $(\nabla, \sigma)$ on $\Omega_{A}^{1}$ descends, due to Assumption 3.6 , to the $B$-bimodule isomorphism

$$
\sigma_{B}: \Omega_{B}^{1} \otimes_{B} \Omega_{B}^{1} \longrightarrow \Omega_{B}^{1} \otimes_{B} \Omega_{B}^{1},[\omega] \otimes_{B}[\zeta] \longmapsto\left[\sigma\left(\omega \otimes_{A} \zeta\right)\right] .
$$


Lemma 3.8. The pair $\left(\nabla_{B}, \sigma_{B}\right)$ defined by 3.15 and 3.16 is a bimodule connection on $\Omega_{B}^{1}$. It reads explicitly as

$$
\nabla_{B}([\omega])=\left[\nabla(\omega)-g^{-1}\left(\omega \otimes_{A} \nu\right) \nabla(\nu)\right]
$$

for all $[\omega] \in \Omega_{B}^{1}$.

Proof. The explicit expression (3.17) is obtained by a short calculation

$$
\begin{aligned}
\nabla_{B}([\omega]) & =\left[\nabla\left(\omega-g^{-1}\left(\omega \otimes_{A} \nu\right) \nu\right)\right]=\left[\nabla(\omega)-g^{-1}\left(\omega \otimes_{A} \nu\right) \nabla(\nu)-\mathrm{d}\left(g^{-1}\left(\omega \otimes_{A} \nu\right)\right) \otimes_{A} \nu\right] \\
& =\left[\nabla(\omega)-g^{-1}\left(\omega \otimes_{A} \nu\right) \nabla(\nu)\right],
\end{aligned}
$$

where in the second step we used the left Leibniz rule for $\nabla$ and in the third step that $\nu$ is identified with 0 in $\Omega_{B}^{1}$ (cf. (3.3)). The left Leibniz rule is a direct consequence of this expression and the right Leibniz rule follows from the fact that $\nabla(\nu) \in \Omega_{A}^{1} \otimes_{A} \Omega_{A}^{1}$ is a central element. The latter statement is proven as follows

$$
a \nabla(\nu)=\nabla(a \nu)-\mathrm{d} a \otimes_{A} \nu=\nabla(\nu a)-\sigma\left(\nu \otimes_{A} \mathrm{~d} a\right)=\nabla(\nu) a,
$$

where we used the left and right Leibniz rules for $(\nabla, \sigma)$, centrality of $\nu$ and Assumption 3.6 .

Remark 3.9. Note that (3.17) is a noncommutative analog of the usual Gauss formula for connections on Riemannian submanifolds, see e.g. [KN96, Chapter VII.3].

In order to prove the main result of this subsection, we require an additional

Assumption 3.10. The diagrams

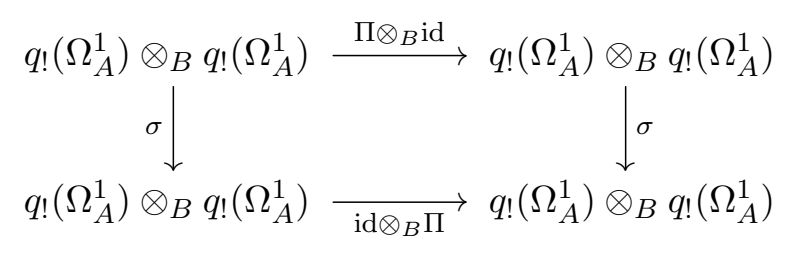

and

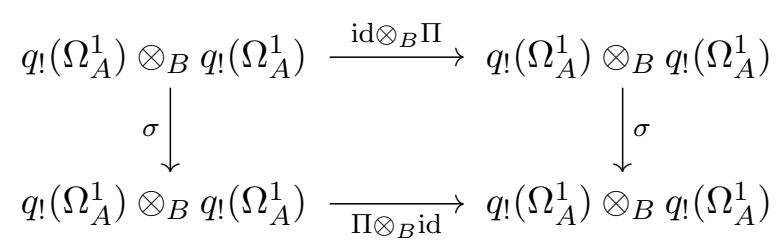

commute.

Proposition 3.11. The pair $\left(g_{B},\left(\nabla_{B}, \sigma_{B}\right)\right)$ defined in (3.9), 3.15) and (3.16) is a Riemannian structure on $\left(\Omega_{B}^{1}, \mathrm{~d}\right)$.

Proof. It remains to prove the symmetry and metric compatibility properties from Definition 2.6 . The symmetry property (2.7) for $\left(g_{B},\left(\nabla_{B}, \sigma_{B}\right)\right)$ follows immediately from Assumption 3.10 and symmetry of $g^{-1}$. To verify metric compatibility 2.8 for $\left(g_{B},\left(\nabla_{B}, \sigma_{B}\right)\right)$, we compute by using metric compatibility of the original Riemannian structure $(g,(\nabla, \sigma))$

$$
\mathrm{d}_{B}\left(g_{B}^{-1}\left([\omega] \otimes_{B}[\zeta]\right)\right)=\left[\left(\mathrm{id} \otimes_{A} g^{-1}\right)\left(\nabla \Pi(\omega) \otimes_{A} \Pi(\zeta)+\sigma_{12}\left(\Pi(\omega) \otimes_{A} \nabla \Pi(\zeta)\right)\right)\right],
$$

where $\sigma_{12}:=\sigma \otimes_{A}$ id. Using Lemma 3.7 (ii), we can in the first term replace $\nabla \Pi(\omega)$ with (id $\otimes_{A}$ $\Pi) \nabla \Pi(\omega)$. Using also Assumption 3.10, we can replace in the second term $\sigma_{12}\left(\Pi(\omega) \otimes_{A} \nabla \Pi(\zeta)\right)$ with (id $\otimes_{A} \Pi \otimes_{A}$ id) $\sigma_{12}\left(\omega \otimes_{A} \nabla \Pi(\zeta)\right)$ and hence via Lemma 3.7 (ii) with (id $\left.\otimes_{A} \Pi \otimes_{A} \Pi\right) \sigma_{12}\left(\omega \otimes_{A}\right.$ $\nabla \Pi(\zeta))$. The resulting expression proves metric compatibility for $\left(g_{B},\left(\nabla_{B}, \sigma_{B}\right)\right)$. 


\subsection{Induced spinorial structure}

We now induce a spinorial structure $\left(\mathcal{E}_{B}, \nabla_{B}^{\mathrm{sp}}, \gamma_{B}\right)$ on the Riemannian structure $\left(g_{B},\left(\nabla_{B}, \sigma_{B}\right)\right)$ from Section 3.2. Our definitions and constructions below are inspired by well-known results on spinorial structures on hypersurfaces in classical differential geometry, see e.g. [Bur93, Tra95, Bar96] and also [HMZ02 for a good review. As the first step, we use the change of base functor (for left modules) to define the $B$-module

$$
\mathcal{E}_{B}:=q_{!}(\mathcal{E}) \cong \frac{\mathcal{E}}{I \mathcal{E}} \in{ }_{B} \operatorname{Mod}
$$

To introduce a suitable Clifford multiplication $\gamma_{B}: \Omega_{B}^{1} \otimes_{B} \mathcal{E}_{B} \rightarrow \mathcal{E}_{B}$, we recall the classical case from [HMZ02, Eqn. (3.4)] and define the $B$-module map

$$
\gamma_{B}: \Omega_{B}^{1} \otimes_{B} \mathcal{E}_{B} \longrightarrow \mathcal{E}_{B},[\omega] \otimes_{B}[s] \longmapsto\left[\gamma_{[2]}\left(\Pi(\omega) \otimes_{A} \nu \otimes_{A} s\right)\right]
$$

where $\gamma_{[2]}$ was defined in $(2.9)$. Note that this map is well-defined since the normalized 1-form $\nu \in \Omega_{A}^{1}$ is central by Definition 3.2 .

The given connection $\nabla^{\text {sp }}: \mathcal{E} \rightarrow \Omega_{A}^{1} \otimes_{A} \mathcal{E}$ on $\mathcal{E} \in{ }_{A}$ Mod descends to a connection $\nabla^{\text {sp }}$ : $\mathcal{E}_{B} \rightarrow \Omega_{B}^{1} \otimes_{B} \mathcal{E}_{B}$ on $\mathcal{E}_{B} \in{ }_{B} \operatorname{Mod}$ because $\left[\nabla^{\mathrm{sp}}(a s)=a \nabla^{\mathrm{sp}}(s)+\mathrm{d} a \otimes_{A} s\right]=0$, for all $a \in I$, as a consequence of the relations in (3.3) and (3.22). This is however not yet the correct induced spin connection on $\mathcal{E}_{B}$. Motivated by the classical spinorial Gauss formula from [HMZ02, Eqn. (3.5)], we define

$$
\nabla_{B}^{\mathrm{sp}}: \mathcal{E}_{B} \longrightarrow \Omega_{B}^{1} \otimes_{B} \mathcal{E}_{B}, \quad[s] \longmapsto\left[\nabla^{\mathrm{sp}}(s)+\frac{1}{2}\left(\mathrm{id} \otimes_{A} \gamma_{[2]}\right)\left(\nabla(\nu) \otimes_{A} \nu \otimes_{A} s\right)\right] .
$$

This defines a connection on the left $B$-module $\mathcal{E}_{B} \in{ }_{B}$ Mod since both $\nu \in \Omega_{A}^{1}$ and $\nabla(\nu) \in$ $\Omega_{A}^{1} \otimes_{A} \Omega_{A}^{1}$ are central. (The latter statement was proven in $(3.19)$.)

In order to prove that these data define a spinorial structure in the sense of Definition 2.8, we require an additional

Assumption 3.12. The element $\nabla(\nu) \in \Omega_{A}^{1} \otimes_{A} \Omega_{A}^{1}$ satisfies

$$
\left[\sigma_{23} \sigma_{12}\left(\Pi(\omega) \otimes_{A} \nabla(\nu)\right)\right]=\left[\nabla(\nu) \otimes_{A} \Pi(\omega)\right] \in \Omega_{B}^{1} \otimes_{B} q_{!}\left(\Omega_{A}^{1}\right) \otimes_{B} q_{!}\left(\Omega_{A}^{1}\right)
$$

for all $\omega \in \Omega_{A}^{1}$, where $\sigma_{12}:=\sigma \otimes_{A}$ id and $\sigma_{23}:=\mathrm{id} \otimes_{A} \sigma$.

Proposition 3.13. The triple $\left(\mathcal{E}_{B}, \nabla_{B}^{\mathrm{sp}}, \gamma_{B}\right)$ defined in (3.22), 3.24) and 3.23) is a spinorial structure on the Riemannian structure $\left(g_{B},\left(\nabla_{B}, \sigma_{B}\right)\right)$.

Proof. It remains to prove the Clifford relations and Clifford compatibility properties from Definition 2.8. In these calculations we frequently use the identities

$$
\left[\gamma_{[2]}\left(\Pi(\omega) \otimes_{A} \nu \otimes_{A} s\right)\right]=-\left[\gamma_{[2]}\left(\nu \otimes_{A} \Pi(\omega) \otimes_{A} s\right)\right]
$$

and

$$
\left[\gamma_{[2]}\left(\nu \otimes_{A} \nu \otimes_{A} s\right)\right]=-[s],
$$

which follow from the Clifford relations 2.10 for $\gamma$, Assumption 3.6, Lemma 3.7 (ii) and the normalization condition 3.6.

The Clifford relations (2.10) for $\gamma_{B}$ follow from a direct calculation, for which we introduce the convenient short notation $\sigma\left(\omega \otimes_{A} \zeta\right)=\sum_{\alpha} \zeta^{\alpha} \otimes_{A} \omega_{\alpha}$. We compute

$$
\begin{aligned}
\gamma_{B[2]}([\omega] & \left.\otimes_{B}[\zeta] \otimes_{B}[s]+\sigma_{B 12}\left([\omega] \otimes_{B}[\zeta] \otimes_{B}[s]\right)\right) \\
& =\left[\gamma_{[4]}\left(\Pi(\omega) \otimes_{A} \nu \otimes_{A} \Pi(\zeta) \otimes_{A} \nu \otimes_{A} s+\sum_{\alpha} \Pi\left(\zeta^{\alpha}\right) \otimes_{A} \nu \otimes_{A} \Pi\left(\omega_{\alpha}\right) \otimes_{A} \nu \otimes_{A} s\right)\right] \\
& =\left[\gamma_{[2]}\left(\Pi(\omega) \otimes_{A} \Pi(\zeta) \otimes_{A} s+\sum_{\alpha} \Pi\left(\zeta^{\alpha}\right) \otimes_{A} \Pi\left(\omega_{\alpha}\right) \otimes_{A} s\right)\right] \\
& =-2 g_{B}^{-1}\left([\omega] \otimes_{B}[\zeta]\right)[s]
\end{aligned}
$$


where in the second step we used (3.26). The last step follows from Assumption 3.10, the Clifford relations for $\gamma$ and the definition of $g_{B}^{-1}$ in $(3.9 \mathrm{~b})$.

Proving the Clifford compatibility property (2.11) for $\nabla_{B}, \nabla_{B}^{\mathrm{sp}}$ and $\gamma_{B}$ is a lengthier computation. Using as above (3.26), Assumption 3.10 and also Clifford compatibility for $\nabla, \nabla^{\text {sp }}$ and $\gamma$, one finds that the desired equality $\nabla_{B}^{\mathrm{sp}} \gamma_{B}\left([\omega] \otimes_{B}[s]\right)=\left(\mathrm{id} \otimes_{B} \gamma_{B}\right)\left(\nabla_{B}^{\otimes}\left([\omega] \otimes_{B}[s]\right)\right)$ is equivalent to the statement that the two expressions

$$
\left[\left(\mathrm{id} \otimes_{A} \gamma_{[2]}\right)\left(\sigma_{12}\left(\Pi(\omega) \otimes_{A} \nabla(\nu) \otimes_{A} s\right)+\frac{1}{2} \nabla(\nu) \otimes_{A} \Pi(\omega) \otimes_{A} s\right)\right]
$$

and

$$
\left[\left(\mathrm{id} \otimes_{A} g^{-1}\right)\left(\nabla \Pi(\omega) \otimes_{A} \nu\right) \otimes_{A} s+\frac{1}{2}\left(\mathrm{id} \otimes_{A} \gamma_{[4]}\right)\left(\sigma_{12} \sigma_{23}\left(\Pi(\omega) \otimes_{A} \nu \otimes_{A} \nabla(\nu) \otimes_{A} \nu_{\otimes_{A}} s\right)\right)\right]
$$

are equal. (The term with $g^{-1}$ in $33.28 \mathrm{~b}$ ) arises from computing (id $\left.\otimes_{A} \Pi\right) \nabla \Pi(\omega)=\nabla \Pi(\omega)-$ $\left(\operatorname{id} \otimes_{A} g^{-1}\right)\left(\nabla \Pi(\omega) \otimes_{A} \nu\right) \otimes_{A} \nu$ via (3.7).) Using metric compatibility (2.8) for $(g,(\nabla, \sigma))$, Lemma 3.7 (ii) and the Clifford relations for $\gamma$, we can rewrite the first term of (3.28b) as

$$
\begin{aligned}
(3.28 \mathrm{~b})^{1 \mathrm{st}} & =\left[-\left(\mathrm{id} \otimes_{A} g^{-1}\right) \sigma_{12}\left(\Pi(\omega) \otimes_{A} \nabla(\nu)\right) \otimes_{A} s\right] \\
& =\left[\frac{1}{2}\left(\mathrm{id} \otimes_{A} \gamma_{[2]}\right)\left(\sigma_{12}\left(\Pi(\omega) \otimes_{A} \nabla(\nu) \otimes_{A} s\right)+\sigma_{23} \sigma_{12}\left(\Pi(\omega) \otimes_{A} \nabla(\nu) \otimes_{A} s\right)\right)\right] .
\end{aligned}
$$

Concerning the second term of (3.28b), we use the Clifford relations for $\gamma$ to bring the left factor of $\nu$ to the right and observe that there is no $g^{-1}$ contribution as a result of Lemma 3.7 (iii). Hence, we can rewrite the second term of $3.28 \mathrm{~b}$ as

$$
(3.28 \mathrm{~b})^{2 \mathrm{nd}}=\left[\frac{1}{2}\left(\mathrm{id} \otimes_{A} \gamma_{[2]}\right)\left(\sigma_{12}\left(\Pi(\omega) \otimes_{A} \nabla(\nu) \otimes_{A} s\right)\right)\right] .
$$

From these simplifications and Assumption 3.12 , it follows that the expressions in $(3.28 \mathrm{~b})$ and (3.28a) are equal. This completes our proof of the Clifford compatibility property.

We conclude this section by presenting an explicit expression for the induced Dirac operator

$$
D_{B}: \mathcal{E}_{B} \stackrel{\nabla_{B}^{\mathrm{sp}}}{\longrightarrow} \Omega_{B}^{1} \otimes_{B} \mathcal{E}_{B} \stackrel{\gamma_{B}}{\longrightarrow} \mathcal{E}_{B}
$$

Proposition 3.14. The induced Dirac operator (3.31) reads explicitly as

$$
D_{B}([s])=\left[-\frac{1}{2}\left(\gamma_{[2]}-\gamma_{[2]}\left(\sigma \otimes_{A} \text { id }\right)\right)\left(\nu \otimes_{A} \nabla^{\mathrm{sp}}(s)\right)+\frac{1}{2} \gamma_{[2]}\left(\left(\Pi \otimes_{A} \mathrm{id}\right) \nabla(\nu) \otimes_{A} s\right)\right],
$$

for all $[s] \in \mathcal{E}_{B}$.

Proof. This is a straightforward calculation using (3.24), (3.23), the projector (3.7) and the Clifford relations (2.10) for $\gamma$, in particular (3.26). Since the relevant steps are similar to those in the proof of Proposition 3.13 , we do not have to write out the details of this calculation.

\section{Examples}

In this section we will illustrate our framework by applying it to the sequence of noncommutative hypersurface embeddings $\mathbb{T}_{\theta}^{2} \hookrightarrow \mathbb{S}_{\theta}^{3} \hookrightarrow \mathbb{R}_{\theta}^{4}$ studied by Arnlind and Norkvist [AN19. We describe first the relevant differential, Riemannian and spinorial structures on the noncommutative embedding space $\mathbb{R}_{\theta}^{4}$ following our definitions in Section 2. We then use our constructions from Section 3 to induce these structures to the noncommutative hypersurface $\mathbb{S}_{\theta}^{3} \hookrightarrow \mathbb{R}_{\theta}^{4}$ and in a second step to the noncommutative hypersurface $\mathbb{T}_{\theta}^{2} \hookrightarrow \mathbb{S}_{\theta}^{3}$. These studies result in explicit expressions for the Dirac operators (in the sense of Definition 2.8) on these noncommutative hypersurfaces. 


\subsection{Noncommutative embedding space $\mathbb{R}_{\theta}^{4}$}

We begin by introducing the noncommutative embedding space $\mathbb{R}_{\theta}^{4}$. Instead of working with real coordinates $x^{\mu}$, for $\mu=1, \ldots, 4$, it will be more convenient to introduce the complex coordinates $z^{1}:=x^{1}+i x^{2}$ and $z^{2}:=x^{3}+i x^{4}$, together with their complex conjugates $z^{3}:=\overline{z^{1}}=x^{1}-i x^{2}$ and $z^{4}:=\overline{z^{2}}=x^{3}-i x^{4}$. The noncommutative embedding space $\mathbb{R}_{\theta}^{4} \cong \mathbb{C}_{\theta}^{2}$ is then described by the noncommutative algebra

$$
A:=\frac{\mathbb{C}\left[z^{1}, z^{2}, z^{3}, z^{4}\right]}{\left(z^{i} z^{j}-R^{j i} z^{j} z^{i}\right)}
$$

that is freely generated by the complex coordinates, modulo the ideal generated by the commutation relations determined by the entries $R^{j i}$ of the matrix

$$
R:=\left(\begin{array}{cccc}
1 & e^{-i \theta} & 1 & e^{i \theta} \\
e^{i \theta} & 1 & e^{-i \theta} & 1 \\
1 & e^{i \theta} & 1 & e^{-i \theta} \\
e^{-i \theta} & 1 & e^{i \theta} & 1
\end{array}\right) \quad, \quad \theta \in \mathbb{R}
$$

For later use, we note that the entries of the matrix $R$ satisfy

$$
R^{i j}=\overline{R^{j i}}
$$

and

$$
R^{i j} R^{j i}=1
$$

where in the latter equation there is no summation over $i$ and $j$.

To define a differential calculus on $A$, let us introduce the free left $A$-module

$$
\Omega_{A}^{1}:=\bigoplus_{i=1}^{4} A \mathrm{~d} z^{i},
$$

which we endow with the right $A$-action determined by

$$
\mathrm{d} z^{i} z^{j}:=R^{j i} z^{j} \mathrm{~d} z^{i}
$$

(Note that this is analogous to the commutation relations in 4.1).) This defines an $A$-bimodule $\Omega_{A}^{1} \in{ }_{A} \operatorname{Mod}_{A}$, which we endow with a differential d : $A \rightarrow \Omega_{A}^{1}$ by setting $\mathrm{d}: z^{i} \mapsto \mathrm{d} z^{i}$ for the generators and extending to all of $A$ via the Leibniz rule.

Proposition 4.1. The pair $\left(\Omega_{A}^{1}, \mathrm{~d}\right)$ is a differential calculus on $A$.

Proof. The statement holds by construction.

The next step is to introduce a Riemannian structure. For this we consider the standard (flat) Euclidean metric on $\mathbb{R}_{\theta}^{4}$, which in complex coordinates reads as

$$
g:=\sum_{i, j=1}^{4} g_{i j} \mathrm{~d} z^{i} \otimes_{A} \mathrm{~d} z^{j} \in \Omega_{A}^{1} \otimes_{A} \Omega_{A}^{1},
$$

where $g_{i j}$ are the entries of the matrix

$$
\left(g_{i j}\right):=\frac{1}{2}\left(\begin{array}{cccc}
0 & 0 & 1 & 0 \\
0 & 0 & 0 & 1 \\
1 & 0 & 0 & 0 \\
0 & 1 & 0 & 0
\end{array}\right)
$$


Using 4.4, 4.3b and 4.2, one easily checks that $g \in \Omega_{A}^{1} \otimes_{A} \Omega_{A}^{1}$ is a central element, hence it defines an $A$-bimodule map $g: A \rightarrow \Omega_{A}^{1} \otimes_{A} \Omega_{A}^{1}$, see Remark 2.5. The inverse metric $g^{-1}$ : $\Omega_{A}^{1} \otimes_{A} \Omega_{A}^{1} \rightarrow A$ is defined on the basis $\left\{\mathrm{d} z^{i} \otimes_{A} \mathrm{~d} z^{j}: i, j=1, \ldots, 4\right\}$ of $\Omega_{A}^{1} \otimes_{A} \Omega_{A}^{1}$ by

$$
g^{-1}\left(\mathrm{~d} z^{i} \otimes_{A} \mathrm{~d} z^{j}\right)=g^{i j},
$$

where $g^{i j}$ are the entries of the matrix

$$
\left(g^{i j}\right)=2\left(\begin{array}{llll}
0 & 0 & 1 & 0 \\
0 & 0 & 0 & 1 \\
1 & 0 & 0 & 0 \\
0 & 1 & 0 & 0
\end{array}\right) .
$$

Observe that

$$
\sum_{j=1}^{4} g_{i j} g^{j k}=\delta_{i}^{k}
$$

Lemma 4.2. The element $g \in \Omega_{A}^{1} \otimes_{A} \Omega_{A}^{1}$ in (4.5) defines a (generalized) metric with inverse metric $g^{-1}: \Omega_{A}^{1} \otimes_{A} \Omega_{A}^{1} \rightarrow A$ defined by (4.6).

Proof. It is sufficient to verify the conditions in Remark 2.5 on the basis $\left\{\mathrm{d} z^{k} \in \Omega_{A}^{1}\right\}$. Using (4.7), we compute

$$
\sum_{i, j=1}^{4} g_{i j} \mathrm{~d} z^{i} g^{-1}\left(\mathrm{~d} z^{j} \otimes_{A} \mathrm{~d} z^{k}\right)=\sum_{i, j=1}^{4} g_{i j} \mathrm{~d} z^{i} g^{j k}=\mathrm{d} z^{k}
$$

The second condition in Remark 2.5 is confirmed through a similar calculation.

We define a connection $\nabla: \Omega_{A}^{1} \rightarrow \Omega_{A}^{1} \otimes_{A} \Omega_{A}^{1}$ on $\Omega_{A}^{1}$ by

$$
\nabla\left(\mathrm{d} z^{i}\right):=0
$$

and the left Leibniz rule. Furthermore, we define an $A$-bimodule isomorphism $\sigma: \Omega_{A}^{1} \otimes_{A} \Omega_{A}^{1} \rightarrow$ $\Omega_{A}^{1} \otimes_{A} \Omega_{A}^{1}$ by

$$
\sigma\left(\mathrm{d} z^{i} \otimes_{A} \mathrm{~d} z^{j}\right):=R^{j i} \mathrm{~d} z^{j} \otimes_{A} \mathrm{~d} z^{i}
$$

and left $A$-linear extension to all of $\Omega_{A}^{1} \otimes_{A} \Omega_{A}^{1}$. (Note that this is analogous to the commutation relations in (4.1).)

Lemma 4.3. The pair $(\nabla, \sigma)$ introduced in 4.9$)$ and 4.10 defines a bimodule connection.

Proof. It remains to confirm the right Leibniz rule from Definition 2.2 (ii). For this it is sufficient to consider homogeneous elements $a=z^{j_{1}} \cdots z^{j_{n}} \in A$, for some $n \in \mathbb{Z}_{\geq 0}$. We compute

$$
\begin{aligned}
\nabla\left(\mathrm{d} z^{i} z^{j_{1}} \cdots z^{j_{n}}\right) & =\nabla\left(R^{j_{1} i} \cdots R^{j_{n} i} z^{j_{1}} \cdots z^{j_{n}} \mathrm{~d} z^{i}\right) \\
& =R^{j_{1} i} \cdots R^{j_{n} i} \mathrm{~d}\left(z^{j_{1}} \cdots z^{j_{n}}\right) \otimes_{A} \mathrm{~d} z^{i} \\
& =\sigma\left(\mathrm{d} z^{i} \otimes_{A} \mathrm{~d}\left(z^{j_{1}} \cdots z^{j_{n}}\right)\right)
\end{aligned}
$$

where in the first equality we used (4.4) and in the second equality we used the left Leibniz rule for the connection (4.9). The last equality follows by using the Leibniz rule to write $\mathrm{d}\left(z^{j_{1}} \cdots z^{j_{n}}\right)=$ $\sum_{k=1}^{n} z^{j_{1}} \cdots z^{j_{k-1} \mathrm{~d}} z^{j_{k}} z^{j_{k+1}} \cdots z^{j_{n}}$ and then using (4.4), (4.3b) and the definition of $\sigma$ in (4.10) in order to rearrange these terms.

Proposition 4.4. The pair $(g,(\nabla, \sigma))$ defined above is a Riemannian structure on $\left(\Omega_{A}^{1}, \mathrm{~d}\right)$. 
Proof. We have to verify the two properties of Definition 2.6. The symmetry property (2.7) is immediate from the definition of $g^{-1}$ in (4.6), $\sigma$ in $(4.10)$ and $R$ in 4.2 . The metric compatibility property (2.8) also holds since

$$
\left(\mathrm{id} \otimes_{A} g^{-1}\right) \nabla^{\otimes}\left(\mathrm{d} z^{i} \otimes_{A} \mathrm{~d} z^{j}\right)=0=\mathrm{d}\left(g^{-1}\left(\mathrm{~d} z^{i} \otimes_{A} \mathrm{~d} z^{j}\right)\right) \otimes_{A} 1,
$$

where the first equality follows from (4.9) and the second equality from $g^{-1}\left(\mathrm{~d} z^{i} \otimes_{A} \mathrm{~d} z^{j}\right) \in \mathbb{C}$.

The final step is to introduce a spinorial structure. For the spinor module, we take the 4-dimensional free left $A$-module

$$
\mathcal{E}:=A^{4} \in{ }_{A} \operatorname{Mod} .
$$

We denote by $\left\{e_{\alpha} \in \mathcal{E}: \alpha=1, \ldots, 4\right\}$ the standard basis for $A^{4}$, i.e. $e_{\alpha}$ is the vector with 1 in the entry $\alpha$ and 0 elsewhere. We define a spin connection $\nabla^{\mathrm{sp}}: \mathcal{E} \rightarrow \Omega_{A}^{1} \otimes_{A} \mathcal{E}$ by

$$
\nabla^{\mathrm{sp}}\left(e_{\alpha}\right):=0
$$

and the left Leibniz rule. Introducing a Clifford multiplication requires some preparations. First, let us recall that the standard Euclidean gamma matrices in Cartesian coordinates on $\mathbb{R}^{4}$ can be expressed in terms of the $2 \times 2$ identity matrix $I_{2}$ and the three Pauli matrices

$$
\sigma^{1}=\left(\begin{array}{ll}
0 & 1 \\
1 & 0
\end{array}\right) \quad, \quad \sigma^{2}=\left(\begin{array}{cc}
0 & -i \\
i & 0
\end{array}\right) \quad, \quad \sigma^{3}=\left(\begin{array}{cc}
1 & 0 \\
0 & -1
\end{array}\right) \quad .
$$

Transforming the standard gamma matrices from Cartesian coordinates to our complex coordinates $z^{i}$, we obtain

$$
\begin{array}{ll}
\gamma^{1}=\left(\begin{array}{cc}
0 & -\sigma^{1}-i \sigma^{2} \\
\sigma^{1}+i \sigma^{2} & 0
\end{array}\right) & , \quad \gamma^{2}=\left(\begin{array}{cc}
0 & -\sigma^{3}-I_{2} \\
\sigma^{3}-I_{2} & 0
\end{array}\right), \\
\gamma^{3}=\left(\begin{array}{cc}
0 & -\sigma^{1}+i \sigma^{2} \\
\sigma^{1}-i \sigma^{2} & 0
\end{array}\right) & ,
\end{array}
$$

By construction, these gamma matrices satisfy the Clifford relations $\left\{\gamma^{i}, \gamma^{j}\right\}:=\gamma^{i} \gamma^{j}+\gamma^{j} \gamma^{i}=$ $-2 g^{i j} I_{4}$, with $g^{i j}$ given in (4.6). These gamma matrices are however not directly applicable to our noncommutative space $\mathbb{R}_{\theta}^{4}$, because the noncommutative Clifford relations (2.10) are given by an anticommutator involving the isomorphism $\sigma$ in 4.10$)$. To address this issue, we introduce the deformed gamma matrices

$$
\begin{array}{ll}
\gamma_{\theta}^{1}=\left(\begin{array}{cc}
0 & e^{\frac{i}{4} \theta}\left(-\sigma^{1}-i \sigma^{2}\right) \\
e^{-\frac{i}{4} \theta}\left(\sigma^{1}+i \sigma^{2}\right) & 0
\end{array}\right), & \gamma_{\theta}^{2}=\left(\begin{array}{cc}
0 & e^{-\frac{i}{4} \theta}\left(-\sigma^{3}-I_{2}\right) \\
e^{\frac{i}{4} \theta}\left(\sigma^{3}-I_{2}\right) & 0
\end{array}\right), \\
\gamma_{\theta}^{3}=\left(\begin{array}{cc}
0 & e^{\frac{i}{4} \theta}\left(-\sigma^{1}+i \sigma^{2}\right) \\
e^{-\frac{i}{4} \theta}\left(\sigma^{1}-i \sigma^{2}\right) & 0
\end{array}\right), \quad \gamma_{\theta}^{4}=\left(\begin{array}{cc}
0 & e^{-\frac{i}{4} \theta}\left(-\sigma^{3}+I_{2}\right) \\
e^{\frac{i}{4} \theta}\left(\sigma^{3}+I_{2}\right) & 0
\end{array}\right),
\end{array}
$$

which can be obtained from the cocycle deformation techniques developed in BLvS13, AS14, BSS14. We define the associated Clifford multiplication $\gamma: \Omega_{A}^{1} \otimes_{A} \mathcal{E} \rightarrow \mathcal{E}$ by

$$
\gamma\left(\mathrm{d} z^{i} \otimes_{A} e_{\alpha}\right):=\gamma_{\theta}^{i} e_{\alpha}
$$

and left $A$-linear extension to all of $\Omega_{A}^{1} \otimes_{A} \mathcal{E}$, where $\gamma_{\theta}^{i} e_{\alpha}$ denotes the action of the matrix $\gamma_{\theta}^{i}$ on the basis spinors $e_{\alpha} \in \mathcal{E}=A^{4}$. Let us record some useful identities that we will use later.

Lemma 4.5. Define the $\theta$-anticommutator $\left\{\gamma_{\theta}^{i}, \gamma_{\theta}^{j}\right\}_{\theta}:=\gamma_{\theta}^{i} \gamma_{\theta}^{j}+R^{j i} \gamma_{\theta}^{j} \gamma_{\theta}^{i}$ and the $\theta$-commutator $\left[\gamma_{\theta}^{i}, \gamma_{\theta}^{j}\right]_{\theta}:=\gamma_{\theta}^{i} \gamma_{\theta}^{j}-R^{j i} \gamma_{\theta}^{j} \gamma_{\theta}^{i}$. Then the following properties hold true: 
(i) $\left\{\gamma_{\theta}^{i}, \gamma_{\theta}^{j}\right\}_{\theta}=R^{j i}\left\{\gamma_{\theta}^{j}, \gamma_{\theta}^{i}\right\}_{\theta}$

(ii) $\left[\gamma_{\theta}^{i}, \gamma_{\theta}^{j}\right]_{\theta}=-R^{j i}\left[\gamma_{\theta}^{j}, \gamma_{\theta}^{i}\right]_{\theta}$

(iii) $\left\{\gamma_{\theta}^{i}, \gamma_{\theta}^{j}\right\}_{\theta}=-2 g^{i j} I_{4}$

Proof. Items (i) and (ii) follow directly from the definitions and 4.3b). Item (iii) is a straightforward calculation.

Proposition 4.6. The triple $\left(\mathcal{E}, \nabla^{\mathrm{sp}}, \gamma\right)$ defined in 4.13, (4.14) and 4.18) is a spinorial structure on the Riemannian structure $(g,(\nabla, \sigma))$.

Proof. We have to verify the two properties of Definition 2.8. The Clifford relations 2.10) follow directly from Lemma 4.5 (iii), because

$$
\begin{aligned}
\left(\gamma_{[2]}+\gamma_{[2]}\left(\sigma \otimes_{A} \mathrm{id}\right)\right)\left(\mathrm{d} z^{i} \otimes_{A} \mathrm{~d} z^{j} \otimes_{A} e_{\alpha}\right) & =\left(\gamma_{\theta}^{i} \gamma_{\theta}^{j}+R^{j i} \gamma_{\theta}^{j} \gamma_{\theta}^{i}\right) e_{\alpha}=\left\{\gamma_{\theta}^{i}, \gamma_{\theta}^{j}\right\}_{\theta} e_{\alpha} \\
& =-2 g^{i j} e_{\alpha}=-2 g\left(\mathrm{~d} z^{i} \otimes_{A} \mathrm{~d} z^{j}\right) e_{\alpha}
\end{aligned}
$$

Clifford compatibility (2.11) follows from

$$
\left(\mathrm{id} \otimes_{A} \gamma\right) \nabla^{\otimes}\left(\mathrm{d} z^{i} \otimes_{A} e_{\alpha}\right)=0=\nabla^{\mathrm{sp}} \gamma\left(\mathrm{d} z^{i} \otimes_{A} e_{\alpha}\right),
$$

where we used (4.9), 4.14) and 4.18).

We can now compute the Dirac operator 2.12 associated with our spinorial structure on $\mathbb{R}_{\theta}^{4}$. Expressing elements $s=\sum_{\alpha=1}^{4} s^{\alpha} e_{\alpha} \in \mathcal{E}$ in terms of our basis and introducing the notation $\mathrm{d} a=: \sum_{i=1}^{4} \partial_{i} a \mathrm{~d} z^{i}$, for all $a \in A$, we obtain

$$
\begin{aligned}
D(s) & =\gamma\left(\nabla^{\mathrm{sp}}(s)\right)=\sum_{\alpha=1}^{4} \gamma\left(\mathrm{d} s^{\alpha} \otimes_{A} e_{\alpha}\right)=\sum_{\alpha=1}^{4} \sum_{i=1}^{4} \partial_{i} s^{\alpha} \gamma_{\theta}^{i} e_{\alpha} \\
& =\sum_{\alpha=1}^{4} \sum_{i=1}^{4} \gamma_{\theta}^{i} \partial_{i} s^{\alpha} e_{\alpha}=\sum_{i=1}^{4} \gamma_{\theta}^{i} \partial_{i} s \quad
\end{aligned}
$$

where in the last equality we used the shorthand notation $\partial_{i} s:=\sum_{\alpha=1}^{4} \partial_{i} s^{\alpha} e_{\alpha}$.

\subsection{Noncommutative hypersurface $\mathbb{S}_{\theta}^{3} \hookrightarrow \mathbb{R}_{\theta}^{4}$}

We now apply our construction from Section 3 to induce the differential, Riemannian and spinorial structure on $\mathbb{R}_{\theta}^{4}$ to the noncommutative 3 -sphere $\mathbb{S}_{\theta}^{3} \hookrightarrow \mathbb{R}_{\theta}^{4}$. This amounts to verifying that this example is a noncommutative hypersurface in the sense of Definition 3.2 and that the Assumptions 3.6, 3.10 and 3.12 for our general construction hold true. We shall also provide explicit expressions for these induced structures and in particular for the induced Dirac operator. To simplify our notation, we will suppress in what follows the square brackets denoting equivalence classes. It will be clear from the context and our general construction in Section 3 which of the following expressions are considered in quotient spaces.

The algebra $B=B_{\mathbb{S}_{\theta}^{3}}$ of the noncommutative Connes-Landi 3-sphere [CL01, CD-V02] is defined as the quotient

$$
B:=A /(f)
$$

of the algebra $A=A_{\mathbb{R}_{\theta}^{4}}$ of $\mathbb{R}_{\theta}^{4}$ (cf. 4.1) ) by the ideal generated by the unit sphere relation

$$
f:=\frac{1}{2}\left(\sum_{i, j=1}^{4} g_{i j} z^{i} z^{j}-1\right)=\frac{1}{2}\left(z^{1} \overline{z^{1}}+z^{2} \overline{z^{2}}-1\right)
$$


where the prefactor $\frac{1}{2}$ is a convenient normalization for the generator of the ideal $(f) \subset A$ that is chosen to match the requirements of Example 3.4. From the commutation relations given by (4.1) and 4.2), one checks that $f \in \mathcal{Z}(A) \subseteq A$ is central.

Proposition 4.7. The 1-form

$$
\nu:=\mathrm{d} f=\sum_{i, j=1}^{4} g_{i j} z^{i} \mathrm{~d} z^{j} \in \Omega_{A}^{1}
$$

is central and normalized. Hence, by Example 3.4, $B=B_{\mathbb{S}_{\theta}^{3}}$ is a noncommutative hypersurface of $A=A_{\mathbb{R}_{\theta}^{4}}$ in the sense of Definition 3.2. The projector $\Pi: q_{!}\left(\Omega_{A}^{1}\right) \rightarrow q_{!}\left(\Omega_{A}^{1}\right)$ from Proposition 3.5 reads explicitly as

$$
\Pi\left(\mathrm{d} z^{i}\right)=\mathrm{d} z^{i}-z^{i} \nu
$$

Proof. Centrality of $\nu$ is a simple check using (4.4) and (4.2) and the normalization condition (3.6) is proven by

$$
g^{-1}\left(\nu \otimes_{A} \nu\right)=\sum_{i, j, k, l=1}^{4} g_{i j} z^{i} g^{j l} g_{k l} z^{k}=\sum_{i, k=1}^{4} g_{i k} z^{i} z^{k}=1 .
$$

The explicit expression for the projector is obtained from a short calculation

$$
\Pi\left(\mathrm{d} z^{i}\right)=\mathrm{d} z^{i}-g^{-1}\left(\mathrm{~d} z^{i} \otimes_{A} \sum_{j, k=1}^{4} g_{j k} z^{j} \mathrm{~d} z^{k}\right) \nu=\mathrm{d} z^{i}-\sum_{j, k=1}^{4} g^{i k} g_{j k} z^{j} \nu=\mathrm{d} z^{i}-z^{i} \nu
$$

where in the second step we used (4.4), 4.2 and 4.6) in order to write $\sum_{j, k=1}^{4} g_{j k} z^{j} \mathrm{~d} z^{k}=$ $\sum_{j, k=1}^{4} \mathrm{~d} z^{k} g_{j k} z^{j}$.

Proposition 4.8. Assumptions 3.6 and 3.10 hold true. The induced Riemannian structure from Proposition 3.11 reads explicitly as

$$
\begin{gathered}
g_{B}=\sum_{i, j=1}^{4} g_{i j} \mathrm{~d} z^{i} \otimes_{B} \mathrm{~d} z^{j} \in \Omega_{B}^{1} \otimes_{B} \Omega_{B}^{1} \\
g_{B}^{-1}\left(\mathrm{~d} z^{i} \otimes_{B} \mathrm{~d} z^{j}\right)=g^{i j}-z^{i} z^{j} \\
\nabla_{B}\left(\mathrm{~d} z^{i}\right)=-z^{i} \sum_{k, l=1}^{4} g_{k l} \mathrm{~d} z^{k} \otimes_{B} \mathrm{~d} z^{l} \\
\sigma_{B}\left(\mathrm{~d} z^{i} \otimes_{B} \mathrm{~d} z^{j}\right)=R^{j i} \mathrm{~d} z^{j} \otimes_{B} \mathrm{~d} z^{i} .
\end{gathered}
$$

Proof. Verifying Assumption 3.6 is a simple check using (4.4), (4.10) and (4.2). To prove commutativity of the top diagram in Assumption 3.10, we use (4.25) and compute

$$
\begin{aligned}
\sigma\left(\Pi\left(\mathrm{d} z^{i}\right) \otimes_{A} \mathrm{~d} z^{j}\right) & =\sigma\left(\mathrm{d} z^{i} \otimes_{A} \mathrm{~d} z^{j}\right)-\sigma\left(z^{i} \nu \otimes_{A} \mathrm{~d} z^{j}\right) \\
& =R^{j i} \mathrm{~d} z^{j} \otimes_{A} \mathrm{~d} z^{i}-R^{j i} \mathrm{~d} z^{j} \otimes_{A} z^{i} \nu \\
& =R^{j i} \mathrm{~d} z^{j} \otimes_{A} \Pi\left(\mathrm{d} z^{i}\right) \\
& =\left(\mathrm{id} \otimes_{A} \Pi\right) \sigma\left(\mathrm{d} z^{i} \otimes_{A} \mathrm{~d} z^{j}\right)
\end{aligned}
$$


where in the second step we used also (3.10) and 4.4). Commutativity of the bottom diagram in Assumption 3.10 is proven by a similar calculation.

Concerning the explicit expressions for the induced Riemannian structure, we observe that 4.28a follows trivially from (3.9a). Equation 4.28b) follows from (3.9b), 4.25) and a straightforward calculation. Equation (4.28c) follows from (3.17) and 4.9 by a short calculation

$$
\nabla_{B}\left(\mathrm{~d} z^{i}\right)=\nabla\left(\mathrm{d} z^{i}\right)-g^{-1}\left(\mathrm{~d} z^{i} \otimes_{A} \nu\right) \nabla(\nu)=-z^{i} \nabla(\nu)=-z^{i} \sum_{k, l=1}^{4} g_{k l} \mathrm{~d} z^{k} \otimes_{B} \mathrm{~d} z^{l}
$$

where in the last step we used that

$$
\nabla(\nu)=\sum_{k, l=1}^{4} g_{k l} \nabla\left(z^{k} \mathrm{~d} z^{l}\right)=\sum_{k, l=1}^{4} g_{k l} \mathrm{~d} z^{k} \otimes_{A} \mathrm{~d} z^{l}
$$

via the left Leibniz rule and (4.9). Finally, (4.28d) follows trivially from (3.16) and 4.10).

Proposition 4.9. Assumption 3.12 holds true. The induced spinorial structure from Proposition 3.13 reads explicitly as

$$
\begin{gathered}
\mathcal{E}_{B}=\frac{\mathcal{E}}{f \mathcal{E}}, \\
\gamma_{B}\left(\mathrm{~d} z^{i} \otimes_{B} e_{\alpha}\right)=-\left(\sum_{k, l=1}^{4} g_{k l} z^{k} \gamma_{\theta}^{l} \gamma_{\theta}^{i}+z^{i}\right) e_{\alpha}, \\
\nabla_{B}^{\mathrm{sp}}\left(e_{\alpha}\right)=\frac{1}{2} \sum_{i, j, k, l=1}^{4} g_{i j} g_{k l} z^{k} \mathrm{~d} z^{i} \otimes_{B} \gamma_{\theta}^{j} \gamma_{\theta}^{l} e_{\alpha} .
\end{gathered}
$$

Proof. Recalling (4.31), Assumption 3.12 is verified by a similar calculation as the one that proves centrality of the metric $g$.

Concerning the explicit expressions for the induced spinorial structure, we observe that 4.32a is just the definition in (3.22). Equation (4.32b) follows from $(3.23)$ by a short calculation

$$
\begin{aligned}
\gamma_{B}\left(\mathrm{~d} z^{i} \otimes_{B} e_{\alpha}\right) & =-\gamma_{[2]}\left(\nu \otimes_{A} \Pi\left(\mathrm{d} z^{i}\right) \otimes_{A} e_{\alpha}\right) \\
& =-\gamma_{[2]}\left(\nu \otimes_{A} \mathrm{~d} z^{i} \otimes_{A} e_{\alpha}\right)+g^{-1}\left(\mathrm{~d} z^{i} \otimes_{A} \nu\right) \gamma_{[2]}\left(\nu \otimes_{A} \nu \otimes_{A} e_{\alpha}\right) \\
& =-\left(\sum_{k, l=1}^{4} g_{k l} z^{k} \gamma_{\theta}^{l} \gamma_{\theta}^{i}+z^{i}\right) e_{\alpha}
\end{aligned}
$$

where in the first step we used (3.26a) and in the third step we used (3.26b). Finally, equation 4.32c follows from writing out (3.24) and using 4.14 and 4.31).

We now have all the building blocks for computing the induced Dirac operator on $\mathbb{S}_{\theta}^{3}$.

Proposition 4.10. The induced Dirac operator 3.31 on $\mathbb{S}_{\theta}^{3}$ is given by

$$
D_{B}(s)=-\frac{1}{2} \sum_{i, j=1}^{4}\left[\gamma_{\theta}^{j}, \gamma_{\theta}^{i}\right]_{\theta} \partial_{i} s z_{j}-\frac{3}{2} s
$$

where $z_{i}:=\sum_{k=1}^{4} g_{i k} z^{k}, \partial_{i} s:=\sum_{\alpha=1}^{4} \partial_{i} s^{\alpha} e_{\alpha}$ and $\left[\gamma_{\theta}^{j}, \gamma_{\theta}^{i}\right]_{\theta}$ is the $\theta$-commutator from Lemma 4.5 . 
Proof. We have to compute the induced Dirac operator from Proposition 3.14 for our example. Using (4.9) and (4.24), the first term of $(3.32)$ is given by

$$
3.32)^{1 \mathrm{st}}=-\frac{1}{2} \sum_{\alpha=1}^{4} \sum_{i, j, k=1}^{4} \partial_{i} s^{\alpha} g_{k j} z^{k}\left(\gamma_{\theta}^{j} \gamma_{\theta}^{i}-R^{i j} \gamma_{\theta}^{i} \gamma_{\theta}^{j}\right) e_{\alpha}=-\frac{1}{2} \sum_{i, j=1}^{4}\left[\gamma_{\theta}^{j}, \gamma_{\theta}^{i}\right]_{\theta} \partial_{i} s z_{j}
$$

which yields the first term of 4.34 . To compute the second term of 3.32 , we first observe that

$$
\left(\Pi \otimes_{A} \mathrm{id}\right) \nabla(\nu)=\sum_{i, j=1}^{4} g_{i j} \Pi\left(\mathrm{d} z^{i}\right) \otimes_{A} \mathrm{~d} z^{j}=\sum_{i, j=1}^{4}\left(g_{i j}-z_{j} z_{i}\right) \mathrm{d} z^{i} \otimes_{A} \mathrm{~d} z^{j},
$$

where in the first step we used (4.31) and in the second step 4.25). This element is invariant under applying $\sigma$, i.e. $\sigma\left(\Pi \otimes_{A}\right.$ id $) \nabla(\nu)=\left(\Pi \otimes_{A}\right.$ id $) \nabla(\nu)$, hence we can write

$$
\left(\Pi \otimes_{A} \mathrm{id}\right) \nabla(\nu)=\frac{1}{2}\left(\left(\Pi \otimes_{A} \mathrm{id}\right) \nabla(\nu)+\sigma\left(\Pi \otimes_{A} \mathrm{id}\right) \nabla(\nu)\right)
$$

in the second term of 3.32 . Using the Clifford relations 2.10, we obtain

$$
(3.32)^{2 \text { nd }}=-\frac{1}{2} \sum_{i, j=1}^{4}\left(g_{i j}-z_{j} z_{i}\right) g^{i j} s=-\frac{1}{2}(4-1) s=-\frac{3}{2} s,
$$

where in the second step we used $\sum_{i, j=1}^{4} g_{i j} g^{i j}=\sum_{i=1}^{4} \delta_{i}^{i}=4$ (cf. 4.7) ) and the sphere relation $\sum_{i, j=1}^{4} z_{j} z_{i} g^{i j}=\sum_{i, j=1}^{4} g_{i j} z^{i} z^{j}=1$ (cf. 4.23)).

Remark 4.11. For vanishing deformation parameter $\theta=0$, our Dirac operator 4.34) on $\mathbb{S}_{\theta}^{3}$ reduces to the usual Dirac operator on the commutative 3 -sphere $\mathbb{S}^{3} \subseteq \mathbb{R}^{4}$, see e.g. [Tra95, Section 7.1] or [Tra93].

We shall now compare our noncommutative hypersurface Dirac operator 4.34 on $\mathbb{S}_{\theta}^{3}$ to the Connes-Landi Dirac operator CL01, CD-V02 that is obtained from an isospectral deformation [BLvS13. This requires some preliminaries on Hopf algebras, their coactions and 2-cocycle deformations, for which we follow the notations and conventions of [BSS17]. Let $H=\mathcal{O}\left(\mathbb{T}^{2}\right)$ denote the Hopf algebra of functions on the 2-torus. A vector space basis for $H$ is given by $\left\{t_{\left(n_{1}, n_{2}\right)}:\left(n_{1}, n_{2}\right) \in \mathbb{Z}^{2}\right\}$, where $t_{\left(n_{1}, n_{2}\right)}$ denotes the exponential function $e^{i\left(n_{1} \phi_{1}+n_{2} \phi_{2}\right)}$ with momentum $\left(n_{1}, n_{2}\right)$. Consider now the left $H$-coaction $\rho: A_{\mathbb{R}^{4}} \rightarrow H \otimes A_{\mathbb{R}^{4}}$ of the torus Hopf algebra on the commutative algebra of functions on $\mathbb{R}^{4}$ that is given in complex coordinates by

$$
\rho\left(z^{1}\right)=t_{(2,0)} \otimes z^{1}, \quad \rho\left(z^{2}\right)=t_{(0,2)} \otimes z^{2}, \quad \rho\left(z^{3}\right)=t_{(-2,0)} \otimes z^{3}, \quad \rho\left(z^{4}\right)=t_{(0,-2)} \otimes z^{4} .
$$

When expressed in terms of the Cartesian coordinates $x^{\mu}$, it is easy to see that this describes (double covers of) rotations in the $\left(x^{1}, x^{2}\right)$-plane and rotations in the $\left(x^{3}, x^{4}\right)$-plane. The noncommutative algebra $A_{\mathbb{R}_{\theta}^{4}}$ given in (4.1) can be obtained as a deformation quantization of the commutative algebra $A_{\mathbb{R}^{4}}$ by introducing the star-product

$$
a \star_{\theta} a^{\prime}:=\sigma_{\theta}\left(a_{(-1)} \otimes a_{(-1)}^{\prime}\right) a_{(0)} a_{(0)}^{\prime},
$$

where we used the standard Sweedler notation $\rho(a)=a_{(-1)} \otimes a_{(0)}$ for left coactions. The 2-cocycle $\sigma_{\theta}: H \otimes H \rightarrow \mathbb{C}$ is defined by

$$
\sigma_{\theta}\left(t_{\left(n_{1}, n_{2}\right)} \otimes t_{\left(m_{1}, m_{2}\right)}\right):=\exp \left(\frac{i \theta}{4}\left(n_{1} m_{2}-n_{2} m_{1}\right)\right) .
$$

Similarly, the algebra $B=B_{\mathbb{S}_{\theta}^{3}}$ of the Connes-Landi sphere 4.22 can be obtained as a deformation quantization of the algebra $B_{\mathbb{S}^{3}}$ of the commutative 3 -sphere. 
One can also obtain the module of noncommutative spinors $\mathcal{E}$ on $\mathbb{R}_{\theta}^{4}$ in 4.13 as a deformation quantization of the module of commutative spinors by introducing the left $H$-coaction

$$
\rho\left(e_{1}\right)=t_{(1,1)} \otimes e_{1}, \quad \rho\left(e_{2}\right)=t_{(-1,-1)} \otimes e_{2}, \quad \rho\left(e_{3}\right)=t_{(1,-1)} \otimes e_{3}, \quad \rho\left(e_{4}\right)=t_{(-1,1)} \otimes e_{4}
$$

and the associated star-module structure

$$
a \star_{\theta} s:=\sigma_{\theta}\left(a_{(-1)} \otimes s_{(-1)}\right) a_{(0)} s_{(0)} .
$$

The same is true for the spinor module $\mathcal{E}_{B}$ on $\mathbb{S}_{\theta}^{3}$ given in 4.32a). When expressed in terms of these star-products, our Dirac operator 4.34 on $\mathbb{S}_{\theta}^{3}$ reads as

$$
D_{B}(s)=-\frac{1}{2} \sum_{i, j=1}^{4}\left[\gamma_{\theta}^{j}, \gamma_{\theta}^{i}\right]_{\theta} \partial_{i}^{\theta} s \star_{\theta} z_{j}-\frac{3}{2} s,
$$

where $\partial_{i}^{\theta}$ is defined by $\mathrm{d} a=\partial_{i}^{\theta} a \star_{\theta} \mathrm{d} z^{i}$ with respect to the deformed module structure.

The Connes-Landi Dirac operator $D_{\mathrm{CL}}$ on $\mathbb{S}_{\theta}^{3}$ is given by regarding the classical Dirac operator on $\mathbb{S}^{3}$ as an operator on the deformed spinor module, see CL01, CD-V02, BLvS13] for details. Concretely, it is given by setting the deformation parameter $\theta=0$ in 4.44 , i.e.

$$
D_{\mathrm{CL}}(s)=-\frac{1}{2} \sum_{i, j=1}^{4}\left[\gamma^{j}, \gamma^{i}\right] \partial_{i} s z_{j}-\frac{3}{2} s,
$$

where $\partial_{i}$ is defined by $\mathrm{d} a=\partial_{i} a \mathrm{~d} z^{i}$ with respect to the undeformed module structure. Because $D_{\mathrm{CL}}$ is equivariant under the torus action, it satisfies the following property

$$
D_{\mathrm{CL}}\left(a \star_{\theta} s\right)=a \star_{\theta} D_{\mathrm{CL}}(s)+\gamma_{\mathbb{S}^{3}}\left(\mathrm{~d} a \otimes_{B_{\mathbb{S}_{\theta}^{3}}} s\right)
$$

where $\omega \otimes_{B_{\mathbb{S}_{\theta}^{3}}} s:=\sigma_{\theta}\left(\omega_{(-1)} \otimes s_{(-1)}\right) \omega_{(0)} \otimes_{B_{\mathbb{S}^{3}}} s_{(0)}$ denotes the deformed tensor product and $\gamma_{\mathbb{S}^{3}}$ the classical Clifford multiplication. Observe that this is precisely the same property that our hypersurface Dirac operator $D_{B}$ satisfies by Proposition 2.9. This is because, in the present context of deformation quantization, our noncommutative Clifford multiplication (4.18) coincides by construction with the classical Clifford multiplication regarded as a map on the deformed modules. (The same is true for the induced Clifford multiplication (3.23) on the noncommutative hypersurface $\mathbb{S}_{\theta}^{3}$ because the normalized form $\nu$ in 4.24 is invariant under the torus action.) With these preparations, we can now prove the following comparison result.

Proposition 4.12. The hypersurface Dirac operator (4.44) on $\mathbb{S}_{\theta}^{3}$ coincides with the ConnesLandi Dirac operator $D_{\mathrm{CL}}$.

Proof. Because both $D_{B}$ and $D_{\mathrm{CL}}$ satisfy the same property (4.46), they coincide if and only if $D_{B}\left(e_{\alpha}\right)=D_{\mathrm{CL}}\left(e_{\alpha}\right)$, for all basis spinors $e_{\alpha}$. The latter follows from (4.44) and (4.45) because $D_{B}\left(e_{\alpha}\right)=-\frac{3}{2} e_{\alpha}=D_{\mathrm{CL}}\left(e_{\alpha}\right)$.

\subsection{Noncommutative hypersurface $\mathbb{T}_{\theta}^{2} \hookrightarrow \mathbb{S}_{\theta}^{3}$}

In this section we apply our construction from Section 3 to induce the differential, Riemannian and spinorial structure on $\mathbb{S}_{\theta}^{3}$ (cf. Section 4.2 ) to the noncommutative 2-torus $\mathbb{T}_{\theta}^{2} \hookrightarrow \mathbb{S}_{\theta}^{3}$. In analogy to Section 4.2, this amounts to verifying that this example is a noncommutative hypersurface in the sense of Definition 3.2 and that the Assumptions 3.6, 3.10 and 3.12 for our general construction hold true. We shall also provide explicit expressions for these induced structures and in particular for the induced Dirac operator. We will again suppress in what follows the square brackets denoting equivalence classes in order to simplify our notations. 
Consider the quotient

$$
C:=B /(\widetilde{f})
$$

of the algebra $B=B_{\mathbb{S}_{\theta}^{3}}$ of $\mathbb{S}_{\theta}^{3}($ cf. 4.22$)$ ) by the ideal generated by

$$
\widetilde{f}:=\frac{1}{2}\left(\sum_{i, j=1}^{4} h_{i j} z^{i} z^{j}\right)=\frac{1}{2}\left(z^{1} \overline{z^{1}}-z^{2} \overline{z^{2}}\right),
$$

where $h_{i j}$ are the entries of the matrix

$$
\left(h_{i j}\right):=\frac{1}{2}\left(\begin{array}{cccc}
0 & 0 & 1 & 0 \\
0 & 0 & 0 & -1 \\
1 & 0 & 0 & 0 \\
0 & -1 & 0 & 0
\end{array}\right) .
$$

From the commutation relations given by (4.1) and (4.2), one checks that $\tilde{f} \in \mathcal{Z}(B) \subseteq B$ is central. We denote the quotient map

$$
\widetilde{q}: B \longrightarrow C
$$

by a tilde in order to distinguish it from the quotient map $q: A \rightarrow B$ in Section 4.2. To recognize $C=C_{\mathbb{T}_{\theta}^{2}}$ as the algebra of the noncommutative 2-torus $\mathbb{T}_{\theta}^{2}$, let us recall from 4.22) that $B=A /(f)$, hence $C=A /(f, \widetilde{f})$ is the quotient of the algebra $A=A_{\mathbb{R}_{\theta}^{4}}$ of $\mathbb{R}_{\theta}^{4}$ by the ideal generated by the two relations $f$ and $\tilde{f}$ in 4.23 and (4.48). The usual torus relations for the rescaled coordinates $u:=\sqrt{2} z^{1}$ and $v:=\sqrt{2 z^{2}}$ are then obtained from the linear combinations

$$
2(f+\widetilde{f})=2 z^{1} \overline{z^{1}}-1=u \bar{u}-1 \quad, \quad 2(f-\widetilde{f})=2 z^{2} \overline{z^{2}}-1=v \bar{v}-1 .
$$

Proposition 4.13. The 1-form

$$
\widetilde{\nu}:=\mathrm{d} \widetilde{f}=\sum_{i, j=1}^{4} h_{i j} z^{i} \mathrm{~d} z^{j} \in \Omega_{B}^{1}
$$

is central and normalized. Hence, by Example 3.4. $C=C_{\mathbb{T}_{\theta}^{2}}$ is a noncommutative hypersurface of $B=B_{\mathbb{S}_{\theta}^{3}}$ in the sense of Definition 3.2. The projector $\widetilde{\Pi}: \widetilde{q}_{!}\left(\Omega_{B}^{1}\right) \rightarrow \widetilde{q}_{!}\left(\Omega_{B}^{1}\right)$ from Proposition 3.5 reads explicitly as

$$
\widetilde{\Pi}\left(\mathrm{d} z^{i}\right)=\mathrm{d} z^{i}+(-1)^{i} z^{i} \widetilde{\nu}
$$

Proof. Centrality of $\widetilde{\nu}$ is a simple check using (4.4) and (4.2). To prove the normalization condition, we use (4.28b) and compute

$$
g_{B}^{-1}\left(\widetilde{\nu} \otimes_{B} \widetilde{\nu}\right)=\sum_{i, j, k, l=1}^{4} h_{i j} z^{i}\left(g^{j l}-z^{j} z^{l}\right) h_{k l} z^{k}=\sum_{i, k=1}^{4} g_{i k} z^{i} z^{k}=1,
$$

where in the second step we used 4.48) and the identity

$$
\sum_{j, l=1}^{4} h_{i j} g^{j l} h_{k l}=g_{i k}
$$


and in the last step we used (4.23). The explicit expression for the projector is obtained from a short calculation

$$
\begin{aligned}
\widetilde{\Pi}\left(\mathrm{d} z^{i}\right) & =\mathrm{d} z^{i}-g_{B}^{-1}\left(\mathrm{~d} z^{i} \otimes_{B} \widetilde{\nu}\right) \widetilde{\nu}=\mathrm{d} z^{i}-\sum_{k, l=1}^{4}\left(g^{i l}-z^{i} z^{l}\right) h_{k l} z^{k} \widetilde{\nu} \\
& =\mathrm{d} z^{i}-\sum_{k, l=1}^{4} g^{i l} h_{k l} z^{k} \widetilde{\nu}=\mathrm{d} z^{i}+(-1)^{i} z^{i} \widetilde{\nu}
\end{aligned}
$$

where in the second step we used 4.28b), in the third step we used 4.48 and the last step follows from $\sum_{l=1}^{4} g^{i l} h_{k l}=-(-1)^{i} \delta_{k}^{i}$.

Proposition 4.14. Assumptions 3.6 and 3.10 hold true. The induced Riemannian structure from Proposition 3.11 reads explicitly as

$$
\begin{gathered}
g_{C}=\sum_{i, j=1}^{4} g_{i j} \mathrm{~d} z^{i} \otimes_{C} \mathrm{~d} z^{j} \in \Omega_{C}^{1} \otimes_{C} \Omega_{C}^{1}, \\
g_{C}^{-1}\left(\mathrm{~d} z^{i} \otimes_{C} \mathrm{~d} z^{j}\right)=g^{i j}-\left(1+(-1)^{i}(-1)^{j}\right) z^{i} z^{j}, \\
\nabla_{C}\left(\mathrm{~d} z^{i}\right)=-z^{i} \sum_{k, l=1}^{4}\left(g_{k l}-(-1)^{i} h_{k l}\right) \mathrm{d} z^{k} \otimes_{C} \mathrm{~d} z^{l}, \\
\sigma_{C}\left(\mathrm{~d} z^{i} \otimes_{C} \mathrm{~d} z^{j}\right)=R^{j i} \mathrm{~d} z^{j} \otimes_{C} \mathrm{~d} z^{i} .
\end{gathered}
$$

Proof. Verifying Assumption 3.6 is a simple check using (4.4), (4.10) and (4.2). To prove commutativity of the top diagram in Assumption 3.10, we use (4.53) and compute

$$
\begin{aligned}
\sigma_{B}\left(\widetilde{\Pi}\left(\mathrm{d} z^{i}\right) \otimes_{B} \mathrm{~d} z^{j}\right) & =R^{j i} \mathrm{~d} z^{j} \otimes_{B} \mathrm{~d} z^{i}+(-1)^{i} z^{i} \mathrm{~d} z^{j} \otimes_{B} \widetilde{\nu} \\
& =R^{j i} \mathrm{~d} z^{j} \otimes_{B} \mathrm{~d} z^{i}+R^{j i} \mathrm{~d} z^{j} \otimes_{B}(-1)^{i} z^{i} \widetilde{\nu} \\
& =\left(\mathrm{id} \otimes_{B} \widetilde{\Pi}\right) \sigma_{B}\left(\mathrm{~d} z^{i} \otimes_{B} \mathrm{~d} z^{j}\right),
\end{aligned}
$$

where in the second step we used (4.4). Commutativity of the bottom diagram in Assumption 3.10 is proven by a similar calculation.

We observe that $4.57 \mathrm{a}$ ) follows trivially from $(3.9 \mathrm{a})$ and $4.57 \mathrm{~b}$ ) follows from $(3.9 \mathrm{~b}), 4.53)$ and a straightforward calculation. Equation (4.57c) follows from (3.17), (4.28c) and

$$
\nabla_{B}(\widetilde{\nu})=\sum_{k, l=1}^{4} h_{k l} \mathrm{~d} z^{k} \otimes_{B} \mathrm{~d} z^{l}
$$

by a short calculation. Finally, (4.57d) follows trivially from (3.16) and 4.28d).

Proposition 4.15. Assumption 3.12 holds true. The induced spinorial structure from Proposition 3.13 reads explicitly as

$$
\begin{gathered}
\mathcal{E}_{C}=\frac{\mathcal{E}_{B}}{\widetilde{f} \mathcal{E}_{B}}=\frac{\mathcal{E}}{f \mathcal{E} \cup \widetilde{f} \mathcal{E}} \\
\gamma_{C}\left(\mathrm{~d} z^{i} \otimes_{C} e_{\alpha}\right)=\left(z^{i} \sum_{k, l, m, n=1}^{4} g_{m n} z^{m} h_{k l} z^{k} \gamma_{\theta}^{l} \gamma_{\theta}^{n}-\sum_{k, l=1}^{4} h_{k l} z^{k} \gamma_{\theta}^{l} \gamma_{\theta}^{i}+(-1)^{i} z^{i}\right) e_{\alpha} \\
\nabla_{C}^{\mathrm{sp}}\left(e_{\alpha}\right)=\frac{1}{2} \sum_{i, j, k, l=1}^{4}\left(g_{k l} z^{k} g_{i j} \mathrm{~d} z^{i}+h_{k l} z^{k} h_{i j} \mathrm{~d} z^{i}\right) \otimes_{C} \gamma_{\theta}^{j} \gamma_{\theta}^{l} e_{\alpha}
\end{gathered}
$$


Proof. Recalling (4.59), Assumption 3.12 is verified by a similar calculation as the one that proves centrality of $\widetilde{f}$ given in (4.48). The explicit expressions in 4.60a), 4.60b and 4.60c follow easily from the definitions (cf. (3.22), (3.23) and (3.24) by straightforward calculations. (To obtain 4.60c), one has to recall that $\widetilde{\nu}=\mathrm{d} f=0$ in $\Omega_{C}^{1}$.)

Proposition 4.16. The induced Dirac operator (3.31) on $\mathbb{T}_{\theta}^{2}$ is given by

$$
D_{C}(s)=-\frac{1}{2} \sum_{i, j=1}^{4}\left[\gamma_{\theta}^{j}, \gamma_{\theta}^{i}\right]_{\theta}\left(\partial_{i} s \widetilde{z}_{j}-\sum_{k=1}^{4} \partial_{k} s z^{k} z_{i} \widetilde{z}_{j}-s z_{i} \widetilde{z}_{j}\right)
$$

where $z_{i}:=\sum_{k=1}^{4} g_{i k} z^{k}, \widetilde{z}_{i}:=\sum_{k=1}^{4} h_{i k} z^{k}, \partial_{i} s:=\sum_{\alpha=1}^{4} \partial_{i} s^{\alpha} e_{\alpha}$ and $\left[\gamma_{\theta}^{j}, \gamma_{\theta}^{i}\right]_{\theta}$ is the $\theta$-commutator from Lemma 4.5.

Proof. The proof is a straightforward but slightly lengthy calculation and hence will not be written out in detail.

From our presentation given in (4.61), it is not easy to interpret and understand $D_{C}$ as a Dirac operator on the flat noncommutative torus $\mathbb{T}_{\theta}^{2}$. We will now simplify (4.61) to a form that admits an obvious interpretation. For this it will be useful to introduce the standard generators

$$
u:=\sqrt{2} z^{1} \quad, \quad v:=\sqrt{2} z^{2} \quad, \quad \bar{u}:=\sqrt{2} \overline{z^{1}} \quad, \quad \bar{v}:=\sqrt{2} \overline{z^{2}}
$$

of the algebra $C$ of $\mathbb{T}_{\theta}^{2}$, which satisfy the relations

$$
\bar{u} u=1, \quad \bar{v} v=1, \quad u v=e^{i \theta} v u .
$$

The module $\Omega_{C}^{1}$ of 1 -forms on $C$ is a 2 -dimensional free module with central basis

$$
\mathrm{d} \phi^{1}:=\frac{1}{i} \bar{u} \mathrm{~d} u \quad, \quad \mathrm{~d} \phi^{2}:=\frac{1}{i} \bar{v} \mathrm{~d} v \quad,
$$

where $i \in \mathbb{C}$ denotes the imaginary unit. (Our notation is inspired by thinking of $u=e^{i \phi^{1}}$ and $v=e^{i \phi^{2}}$ as exponential functions.) The inverse metric $4.57 \mathrm{~b}$ in this basis reads as

$$
g_{C}^{-1}\left(\mathrm{~d} \phi^{i} \otimes \mathrm{d} \phi^{j}\right)=2 \delta^{i j},
$$

where the factor 2 is due to the fact that our embedded noncommutative torus $\mathbb{T}_{\theta}^{2} \hookrightarrow \mathbb{S}_{\theta}^{3}$ has radius $\frac{1}{\sqrt{2}}$, see 4.51 . The differential $\mathrm{d} a=\partial_{\phi^{1}} a \mathrm{~d} \phi^{1}+\partial_{\phi^{2}} a \mathrm{~d} \phi^{2}$ of any $a \in C$ can be expressed in the basis (4.63). Comparing this to $\mathrm{d} a=\sum_{i=1}^{4} \partial_{i} a \mathrm{~d} z^{i} \in \Omega_{C}^{1}$, we find

$$
\partial_{1} a=\frac{2}{i} \partial_{\phi^{1}} a \overline{z^{1}} \quad, \quad \partial_{2} a=\frac{2}{i} \partial_{\phi^{2}} a \overline{z^{2}} \quad, \quad \partial_{3} a=0 \quad, \quad \partial_{4} a=0
$$

for the noncommutative partial derivatives along $z^{i}$.

To simplify the induced Dirac operator 4.61) on $\mathbb{T}_{\theta}^{2}$, we use the Clifford relations in the form of Lemma 4.5 (iii) and obtain after a short calculation

$$
D_{C}(s)=-\gamma\left(\widetilde{\nu} \otimes_{C} \sum_{i=1}^{4}\left(\gamma_{\theta}^{i} \partial_{i} s-\gamma_{\theta}^{i} s z_{i}\right)\right)+\gamma_{[2]}\left(\widetilde{\nu} \otimes_{C} \nu \otimes_{C} \sum_{i=1}^{4} \partial_{i} s z^{i}\right)+\sum_{i=1}^{4}(-1)^{i} \partial_{i} s z^{i} .
$$

Applying the map $\gamma\left(\widetilde{\nu} \otimes_{C}(-)\right): \mathcal{E}_{C} \rightarrow \mathcal{E}_{C}$ to this expression, which squares to - id because $\widetilde{\nu}$ is normalized, we define

$$
\begin{aligned}
\widetilde{D}_{C}(s) & :=\gamma\left(\widetilde{\nu} \otimes_{C} D_{C}(s)\right) \\
& =\sum_{i=1}^{4}\left(\gamma_{\theta}^{i} \partial_{i} s-\gamma_{\theta}^{i} s z_{i}\right)-\gamma\left(\nu \otimes_{C} \sum_{i=1}^{4} \partial_{i} s z^{i}\right)+\gamma\left(\widetilde{\nu} \otimes_{C} \sum_{i=1}^{4}(-1)^{i} \partial_{i} s z^{i}\right) .
\end{aligned}
$$


Inserting 4.65, 4.24 and 4.52 into this expression and carrying out all summations, one obtains

$$
\begin{aligned}
\widetilde{D}_{C}(s)=\frac{1}{i}\left(\gamma_{\theta}^{1} \partial_{\phi^{1}} s \overline{z^{1}}-\gamma_{\theta}^{3} \partial_{\phi^{1}} s z^{1}\right)-\frac{1}{2}\left(\gamma_{\theta}^{1} s \overline{z^{1}}+\gamma_{\theta}^{3} s z^{1}\right) \\
+\frac{1}{i}\left(\gamma_{\theta}^{2} \partial_{\phi^{2}} s \overline{z^{2}}-\gamma_{\theta}^{4} \partial_{\phi^{2}} s z^{2}\right)-\frac{1}{2}\left(\gamma_{\theta}^{2} s \overline{z^{2}}+\gamma_{\theta}^{4} s z^{2}\right)
\end{aligned}
$$

Let us introduce the $C$-module map $\widetilde{\gamma}: \Omega_{C}^{1} \otimes_{C} \mathcal{E}_{C} \rightarrow \mathcal{E}_{C}$ by defining

$$
\widetilde{\gamma}\left(\mathrm{d} \phi^{1} \otimes_{C} s\right):=\frac{1}{i}\left(\gamma_{\theta}^{1} s \overline{z^{1}}-\gamma_{\theta}^{3} s z^{1}\right) \quad, \quad \widetilde{\gamma}\left(\mathrm{d} \phi^{2} \otimes_{C} s\right):=\frac{1}{i}\left(\gamma_{\theta}^{2} s \overline{z^{2}}-\gamma_{\theta}^{4} s z^{2}\right),
$$

for all $s \in \mathcal{E}_{C}$. One easily shows that $\widetilde{\gamma}$ satisfies the Clifford relations

$$
\widetilde{\gamma}_{[2]}\left(\mathrm{d} \phi^{i} \otimes_{C} \mathrm{~d} \phi^{j} \otimes_{C} s\right)+\widetilde{\gamma}_{[2]}\left(\mathrm{d} \phi^{j} \otimes_{C} \mathrm{~d} \phi^{i} \otimes_{C} s\right)=-2 g_{C}^{-1}\left(\mathrm{~d} \phi^{i} \otimes \mathrm{d} \phi^{j}\right) s=-4 \delta^{i j} s
$$

for the inverse metric (4.64). (Note that there is no $\sigma$ in this expression because $\sigma\left(\mathrm{d} \phi^{i} \otimes_{C} \mathrm{~d} \phi^{j}\right)=$ $\mathrm{d} \phi^{j} \otimes_{C} \mathrm{~d} \phi^{i}$.) This allows us to write (4.68) as

$$
\begin{aligned}
\widetilde{D}_{C}(s)= & \widetilde{\gamma}\left(\mathrm{d} \phi^{1} \otimes_{C}\left(\partial_{\phi_{1}} s+\frac{1}{4} \widetilde{\gamma}\left(\mathrm{d} \phi^{1} \otimes_{C}\left(\gamma_{\theta}^{1} s \overline{z^{1}}+\gamma_{\theta}^{3} s z^{1}\right)\right)\right)\right) \\
& +\widetilde{\gamma}\left(\mathrm{d} \phi^{2} \otimes_{C}\left(\partial_{\phi_{2}} s+\frac{1}{4} \widetilde{\gamma}\left(\mathrm{d} \phi^{2} \otimes_{C}\left(\gamma_{\theta}^{2} s \overline{z^{2}}+\gamma_{\theta}^{4} s z^{2}\right)\right)\right)\right) \\
= & \widetilde{\gamma}\left(\mathrm{d} \phi^{1} \otimes_{C}\left(\partial_{\phi_{1}} s+\frac{1}{8 i}\left[\gamma_{\theta}^{1}, \gamma_{\theta}^{3}\right]_{\theta} s\right)\right)+\widetilde{\gamma}\left(\mathrm{d} \phi^{2} \otimes_{C}\left(\partial_{\phi_{2}} s+\frac{1}{8 i}\left[\gamma_{\theta}^{2}, \gamma_{\theta}^{4}\right]_{\theta} s\right)\right),
\end{aligned}
$$

which we recognize as the Dirac operator on $\mathbb{T}_{\theta}^{2}$ corresponding to a rotating frame spin structure, see [BG19]. By a direct calculation, one shows that the spectrum of this operator, and hence the spectrum of the Dirac operator $D_{C}$ in 4.61 on the noncommutative torus $\mathbb{T}_{\theta}^{2}$, is given by

$$
\left\{ \pm \sqrt{2} \sqrt{\left(m+\frac{1}{2}\right)^{2}+\left(n+\frac{1}{2}\right)^{2}}: m, n \in \mathbb{Z}\right\} .
$$

We note that this coincides with the spectrum of the Dirac operator corresponding to the $(1,1)$ spin structure on the commutative 2 -torus $\mathbb{T}^{2}$, see e.g. [Fri84]. (The factor $\sqrt{2}$ in 4.72 is because our noncommutative torus $\mathbb{T}_{\theta}^{2} \hookrightarrow \mathbb{S}_{\theta}^{3}$ has radius $\frac{1}{\sqrt{2}}$.)

By the same argument as in Proposition 4.12, which however involves now a considerably lengthier calculation to compute $D_{C}\left(e_{\alpha}\right)$ on the basis spinors, one can show that, when expressed in terms of star-products, our noncommutative hypersurface Dirac operator 4.71) on $\mathbb{T}_{\theta}^{2}$ coincides with the isospectral deformation [BLvS13] of the classical Dirac operator of type $(1,1)$ on the commutative 2-torus, acting as in [BG19] on doubled, i.e. 4-dimensional, spinors.

\section{Acknowledgments}

We would like to thank Branimir Ćaćić for very useful comments on the manuscript and in particular for suggesting Definition 3.2 to us. We would also like to thank Joakim Arnlind, John Barrett, James Gaunt, Shahn Majid and Axel Tiger Norkvist for useful comments related to this work. H.N. is supported by a PhD Scholarship from the School of Mathematical Sciences of the University of Nottingham. A.S. gratefully acknowledges the financial support of the Royal Society (UK) through a Royal Society University Research Fellowship (UF150099), a Research Grant (RG160517) and two Enhancement Awards (RGF $\backslash \mathrm{EA} \backslash 180270$ and RGF $\backslash \mathrm{EA} \backslash 201051$ ). 


\section{References}

[AN19] J. Arnlind and A. T. Norkvist, "Noncommutative minimal embeddings and morphisms of pseudo-Riemannian calculi," arXiv:1906.03885 [math.QA].

[AS14] P. Aschieri and A. Schenkel, "Noncommutative connections on bimodules and Drinfeld twist deformation," Adv. Theor. Math. Phys. 18, no. 3, 513 (2014) [arXiv:1210.0241 [math.QA]].

[Bar96] C. Bär, "Metrics with harmonic spinors," Geom. Funct. Anal. 6, no. 6, 899-942 (1996).

[BSS14] G. E. Barnes, A. Schenkel and R. J. Szabo, "Nonassociative geometry in quasi-Hopf representation categories I: Bimodules and their internal homomorphisms," J. Geom. Phys. 89, 111 (2014) [arXiv:1409.6331 [math.QA]].

[BSS17] G. E. Barnes, A. Schenkel and R. J. Szabo, "Mapping spaces and automorphism groups of toric noncommutative spaces," Lett. Math. Phys. 107, no. 9, 1591-1628 (2017) [arXiv:1606.04775 [math.QA]].

[BG19] J. W. Barrett and J. Gaunt, "Finite spectral triples for the fuzzy torus," arXiv:1908.06796 [math.QA].

[BM17] E. Beggs and S. Majid, "Spectral triples from bimodule connections and Chern connections," J. Noncommut. Geom. 11, no. 2, 669-701 (2017) [arXiv:1508.04808 [math.QA]].

[BM20] E. Beggs and S. Majid, Quantum Riemannian Geometry, Grundlehren der mathematischen Wissenschaften 355, Springer Verlag (2020).

[BLvS13] S. Brain, G. Landi and W. D. van Suijlekom, "Moduli Spaces of Instantons on Toric Noncommutative Manifolds," Adv. Theor. Math. Phys. 17, no. 5, 1129 (2013) [arXiv:1204.2148 [math-ph]].

[Bur93] J. Bureš, "Dirac operators on hypersurfaces," Comment. Math. Univ. Carolin. 34, no. 2, 313-322 (1993).

[Con94] A. Connes, Noncommutative geometry, Academic Press, Inc., San Diego, CA (1994).

[CD-V02] A. Connes and M. Dubois-Violette, "Noncommutative finite dimensional manifolds: Spherical manifolds and related examples," Commun. Math. Phys. 230, 539 (2002) [math/0107070 [math-qa]].

[CL01] A. Connes and G. Landi, "Noncommutative manifolds: The instanton algebra and isospectral deformations," Commun. Math. Phys. 221, 141 (2001) [math/0011194 [math.QA]].

[CM14] A. Connes and H. Moscovici, "Modular curvature for noncommutative two-tori," J. Amer. Math. Soc. 27, no. 3, 639-684 (2014) [arXiv:1110.3500 [math.QA]].

[D-V01] M. Dubois-Violette, "Lectures on graded differential algebras and noncommutative geometry," in: Y. Maeda, H. Moriyoshi, H. Omori, D. Sternheimer, T. Tate and S. Watamura (eds.), Noncommutative differential geometry and its applications to physics, Math. Phys. Stud. 23, 245-306, Kluwer Acad. Publ., Dordrecht (2001) [arXiv:math/9912017 [math.QA]].

[Fri84] T. Friedrich, "Zur Abhängigkeit des Dirac-Operators von der Spin-Struktur," Colloquium Mathematicae 48, vol. 1, 57-62 (1984).

[HMZ02] O. Hijazi, S. Montiel and X. Zhang, "Conformal lower bounds for the Dirac operator of embedded hypersurfaces," Asian J. Math. 6, no. 1, 23-36 (2002). 
[KN96] S. Kobayashi and K. Nomizu, Foundations of differential geometry Vol. II, Wiley Classics Library, John Wiley \& Sons Inc., New York (1996).

[Lan97] G. Landi, An Introduction to noncommutative spaces and their geometry, Lect. Notes Phys. Monogr. 51, 1 (1997) [hep-th/9701078].

[Tra93] A. Trautman, "Spin structures on hypersurfaces and the spectrum of the Dirac operator on spheres," in: Z. Oziewicz, B. Jancewicz, A. Borowiec (eds), Spinors, Twistors, Clifford Algebras and Quantum Deformations, Fundamental Theories of Physics (An International Book Series on The Fundamental Theories of Physics: Their Clarification, Development and Application), vol 52. Springer, Dordrecht (1993).

[Tra95] A. Trautman, "The Dirac operator on hypersurfaces," Acta Phys. Polon. B 26, no. 7, 1283-1310 (1995) [arXiv:hep-th/9810018]. 